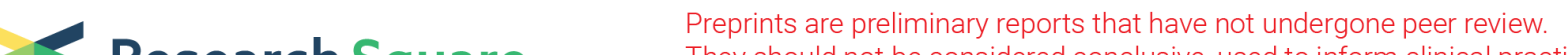 Research Square They should not be considered conclusive, used to inform clinical practice, or referenced by the media as validated information.
}

\section{Gut-brain axis neurodegeneration in a Drosophila model of Parkinson's disease is linked to mitochondrial dysfunction}

\section{Giorgio Fedele}

University Of Cambridge

Samantha Loh

University Of Cambridge

Ivana Celardo

University Of Cambridge

Susann Lehmann

MRC Toxicology Unit

Ana Costa

MRC Toxicology Unit

L. Miguel Martins ( $\triangle$ Im795@cam.ac.uk)

University Of Cambridge https://orcid.org/0000-0002-3019-4809

\section{Article}

Keywords: Drosophila, mitochondria, Parkinson's disease, pink1, Relish, innate immunity, intestine, brain, gut-brain communication

Posted Date: December 16th, 2020

DOl: https://doi.org/10.21203/rs.3.rs-100637/v1

License: (9) This work is licensed under a Creative Commons Attribution 4.0 International License. Read Full License

Version of Record: A version of this preprint was published at Nature Aging on April 4th, 2022. See the published version at https://doi.org/10.1038/s43587-022-00194-z. 


\section{Abstract}

The innate immune response mounts a defence against foreign invaders, but the inappropriate induction of an innate immune response can cause diseases. Previous studies have provided ample evidence showing that mitochondria can be repurposed to promote inflammatory signalling. Damaged mitochondria can also trigger inflammation and promote diseases. Mutations in pink 1 cause early-onset Parkinson's disease (PD), and studies using Drosophila melanogaster have shown that pink1 mutants accumulate damaged mitochondria. Here, we showed that defective mitochondria in pink 1 mutants activate Relish targets and demonstrated that inflammatory signalling causes intestinal dysfunction in pink 1-mutant flies. These effects result in the death of intestinal cells and metabolic reprogramming, which leads to neurotoxicity. We found that Relish signalling is activated downstream of a pathway stimulated by cytosolic DNA. The suppression of Relish in the intestinal midgut of pink1-mutant flies restores mitochondrial function and protects neurons in the brain. We thus conclude that the gut-brain axis causes neurotoxicity in a fly model of PD through a mechanism involving mitochondrial dysfunction.

\section{Introduction}

Animals use the innate immune system as a defence against foreign invaders, which allows a rapid reaction to invading pathogens, such as microbes. Microbes often release foreign nucleic acids into the cytosol of infected cells, which can trigger innate immunity.

Mitochondria are energy-generating organelles that evolved from endosymbionts related to bacteria. Mitochondria retain some of their genetic material in the form of DNA (mtDNA). mtDNA can trigger inflammatory responses similarly to bacterial DNA, and these responses are associated with the absence of methylated $\mathrm{CpG}$ sequences in both bacterial DNA and mtDNA ${ }^{1}$.

Cells safeguard the health of their mitochondria by operating several quality control (QC) mechanisms that ensure the disposal of faulty mitochondria. The organellar QC of organelles involves the degradation of defective mitochondria via mitophagy, which is a form of autophagy (reviewed in ${ }^{2}$ ). Mutations in the gene encoding the mitochondrial kinase PINK1 lead to the accumulation of defective mitochondria and cause a form of familial Parkinson's disease (PD) (reviewed in ${ }^{2}$ ). PINK1 functions in a molecular pathway that ensures the degradation of faulty mitochondria via mitophagy. In mice, the blockage of mitophagy induces the escape of mtDNA into the cytosol, where it activates innate immunity ${ }^{3}$.

In the fruit fly (Drosophila melanogaster), the innate immune system consists of two branches (reviewed in ${ }^{4}$ ): the Toll signalling pathway and the immune deficiency (Imd) pathway. The Imd pathway activates the Rel/NF-kB transcription factor Relish, which controls the expression of several antimicrobial peptides and is indispensable for normal immunity in flies. Relish rewires the metabolism by attenuating FOXOmediated lipolysis ${ }^{5}$ and can also activate programmed cell death ${ }^{6}$. 
The main immune organs in Drosophila are the fat body, which is considered to be equivalent to both vertebrate adipocytes and the liver, and the intestine. The intestine, in particular, plays a key role in activating the Imd pathway (reviewed in ${ }^{7}$ ).

Mounting an immune response is energetically costly and requires trade-offs with other important biological functions (reviewed in ${ }^{8}$ ), and in flies, this trade-off is important during starvation. In such settings, relish mutants exhibit increased survival when deprived of food ${ }^{9}$. The activation of Relish in response to cytosolic DNA can be mediated by interactions with Drosophila Eya, which is a molecule that maintains Relish in the cytoplasm ${ }^{10}$.

Here, we found that in pink 1-mutant flies, in which mitophagy is blocked, Relish was activated with neurotoxic consequences. The accumulation of defective mitochondria in these mutants lead to increased Relish signalling, caused intestinal dysfunction and resulted in cell death in the midgut. Enhanced Relish signalling also resulted in metabolic alterations in pink 1-mutant flies. These alterations are characterized by the accumulation of triglycerides (TAGs) due to the Relish-dependent regulation of lipid catabolism ${ }^{5}$ and the failure of beta oxidation ${ }^{11}$. The genetic suppression of Relish or Eya, a Relishbinding protein involved in the sensing of cytosolic DNA, suppressed neurodegeneration in pink 7-mutant flies. To understand the links between intestinal dysfunction and neurodegeneration, we subsequently investigated whether intestinal dysfunction can prime neurodegeneration and found that the prevention of intestinal dysfunction through either the suppression of Relish expression or the blockage of cell death in the midgut of pink1-mutant flies is sufficient to suppress the central nervous system (CNS) phenotypes of these flies. We conclude that the CNS defects in pink1-mutant flies result from a non-cell autonomous signalling pathway induced by mitochondrial toxicity acting between the intestine and the brain.

\section{Results}

\section{Identification of a Relish transcriptional signature in pink1-mutant flies.}

We previously showed that mitochondrial defects in pink1-mutant flies lead to the upregulation of both nucleotide metabolism and immune response genes ${ }^{11}$. Here, we first explored the mechanism underlying the activation of the innate immune response pathways associated with mitochondrial dysfunction in pink 1-mutant flies. We analysed innate immunity-related transcripts and proteins in pink1-mutant flies through an in silico approach (Fig. 1A) and detected 42 upregulated transcripts that matched a curated list of innate immunity-related genes in flies (Fig. 1B and Supplementary Table 1). Because pink1-mutant flies exhibit a global shutdown of protein synthesis ${ }^{12}$, we also measured the levels of individual proteins in adult flies through quantitative proteomics. We detected the upregulation of nine proteins belonging to the innate immunity pathways in pink1-mutant flies (Fig. 1B and Supplementary Table 2). The transcriptional control of the innate immune response in Drosophila is mediated by signalling cascades that regulate the NF-kB-like transcription factors Dif, Dorsal and Relish (Rel) (reviewed in ${ }^{13}$ ). To identify the upstream regulators of the innate immunity signature present in pink1-mutant flies, we used iRegulon, 
a tool used for the reverse-engineering of transcriptional networks ${ }^{14}$. This analysis identified Rel as the top upstream regulator of the innate immunity signature in pink1-mutant flies (Fig. 1B and Supplementary Table 3).

\section{A relish mutation suppresses the neuronal defects in pink1-mutant flies.}

In flies, mutations in pink1 affect neuronal function, which leads to disruption of circadian rhythms in young adults ${ }^{15}$ by preventing the secretion of neuropeptides ${ }^{16}$ and the selective loss of dopaminergic neurons in the protocerebral posterior lateral 1 (PPL1) cluster in aged flies ${ }^{17}$. We subsequently tested whether mutation of the Relish gene in $\mathrm{Re}^{\mathrm{E} 20 /+ \text { flies }}{ }^{18}$ affected the neuronal phenotype of pink 1 -mutant flies. Specifically, we monitored the locomotor activity during the light-dark cycle (LD) for 7 days and confirmed that pink1-mutant flies show aberrant activity patterns (Fig. 2A). As previously reported ${ }^{19}$, pink1-mutant flies exhibited a significantly longer rest (or inactivity) duration, which was correlated with lower activity levels. Interestingly, the comparison of pink1-mutant flies with pink1,RelE20/+ doublemutant flies revealed that the Relish mutation led to a significant rescue of the sleep-wake patterns observed in pink 1-mutant flies toward those of control flies (Fig. 2A, red and blue). Additionally, the presence of a Relish mutation in the pink 1-mutant flies was sufficient to rescue the loss of DA neurons (Fig. 2B).

The activity of Relish during infection is negatively regulated by calcineurins and protein phosphatases. Calcineurins can be targeted by immunosuppressants, such as tacrolimus, which is used in human organ transplantation to lower the risk of rejection. Calcineurins are present in Drosophila ${ }^{20}$, and we thus tested whether pharmacological intervention with tacrolimus could suppress dopaminergic neurodegeneration in pink 1-mutant flies. The exposure of these mutants to a diet supplemented with tacrolimus prevented the selective loss of dopaminergic neurons in the PPL1 cluster (Fig. 2C). Collectively, these findings show that suppressing innate immunity pathways by manipulating Relish signalling is neuroprotective in a model of pink1 mutation-induced mitochondrial dysfunction.

\section{Intestinal dysfunction in pink1-mutant flies.}

Relish can induce immune responses to pathogens by inducing the transcription of antimicrobial peptides such as Attacin ${ }^{21}$. We subsequently monitored the activation of Relish by expressing a GFP transgene fused to the Attacin-A promoter (Att-GFP) ${ }^{22}$ and detected GFP fluorescence in several regions of the pink1-mutant flies, including their abdominal region (Fig. 3A). A more detailed analysis of the gut showed an increase in GFP expression in the gut enterocytes of pink1-mutant flies (Fig. 3B), which indicated the activation of Relish in the gut. Relish activation involves cleavage of an inhibitory domain on the full-length protein and translocation of the cleaved domain, Rel-68, to the cell nucleus (reviewed in $\left.{ }^{4}\right)$. We subsequently monitored the levels of cytosolic Relish and found lower levels of the full-length protein (Fig. 3C) and increased levels of the Relish transcript (Fig. 3D) in the pink1-mutant flies. Taken together, these results suggest that mitochondrial dysfunction in pink1-mutant flies is associated with Relish activation. 
The increase in the expression of immunity-related transcripts is tightly linked to intestinal barrier dysfunction in Drosophila ${ }^{23}$. We subsequently performed a Smurf assay, which is a non-invasive method for determining the intestinal integrity in adult flies. The Smurf assay showed that pink1-mutant flies exhibit a compromised intestinal barrier that can partially be rescued by a mutation in Relish (Fig. 3E).

In Drosophila, the fat body, which is a lipid storage reservoir, and the gut form the primary immune organs. To visualize the gut and fat body in pink1-mutant flies, we labelled these tissues with phalloidin (to mark the gut) and BODIPY, a fluorescent lipophilic dye that stains neutral lipids. A confocal microscopy analysis of pink 1-mutant flies showed a ring of visceral fat (fat 'doughnut') surrounding the posterior end of the posterior midgut (anterior of the Malpighian tubule junction) (Fig. 4A). Fat body cells migrate to sites of tissue damage, where they clear wound-related cell debris and upregulate antimicrobial peptides (AMPs) ${ }^{22}$. Given their role in cell repair and renewal, we subsequently assessed whether the recruitment of fat body cells to the midgut was associated with damage to the midgut. We therefore assessed the degree of apoptotic cell death in the midgut of pink1-mutant flies and found increased levels of the active Drosophila caspase Dcp-1, an apoptosis effector (Figs. 4B and 4D), which indicated that pink1-mutant flies exhibit increased intestinal damage. Damage to the intestine also results in a robust proliferative response by intestinal stem cells (ISCs) (reviewed in ${ }^{24}$ ). We also monitored the levels of ISC proliferation using a GFP reporter for Escargot (Esg), which is expressed in these cells ${ }^{25}$, and found an increase in the number of Esg-positive cells in pink 1-mutant flies (Figs. 4C and $4 \mathrm{E})$. Taken together, these data show that mitochondrial dysfunction in pink 1 -mutant flies is associated with intestinal damage.

\section{Mutations in eya, a DNA-responsive immune stimulator, suppress the neuronal defects in pink1-mutant flies}

In Drosophila, lipid droplets act as mediators of host-pathogen interactions ${ }^{26}$; thus, their recruitment around the gut in pink1-mutant flies (Fig. 4A) might underlie an infection. Mitochondrial stress in pink1mutant flies causes disruption of the mitochondrial ultrastructure and damage to the mitochondrial membranes in various tissues, such as the indirect flight muscle. In mice, DNA released from mitochondria into the cytosol of cells following mitochondrial stress activates the innate immune response ${ }^{27}$. Because mitochondrial stress can trigger the release of mitochondrial DNA (mtDNA) into the cytosol, we assessed the presence of extramitochondrial DNA in intestinal enterocytes of pink1-mutant flies. A confocal analysis of cells labelled with mitochondria-targeted GFP (mitoGFP) and stained with anti-DNA showed increases in the extramitochondrial DNA levels in pink1-mutant flies (Figs. 5A and 5B).

In Drosophila, the Eya protein acts in a cascade that senses undigested cytosolic DNA and activates the immune response. Eya binds to the inhibitor of nuclear factor kappa-B kinase subunit beta (IKK $\beta)^{10}$ and full-length Relish ${ }^{10,28}$. Because neuronal dysfunction in pink7-mutant flies is suppressed by a Relish mutation, we subsequently tested whether eya can act upstream of Relish in response to naked mtDNA. We noted that the mRNA levels of Relish target genes were decreased in pink1-and-eya double-mutant flies (Fig. 5D). The eya mutation also rescued the defects in activity observed in pink 7 -mutant flies 
(Fig. 5E, red and blue) and the loss of DA neurons (Fig. 5F). We conclude that the neurotoxic consequences of the activation of the immune response in pink1-mutant flies are linked to the presence of extramitochondrial DNA in the cytosol.

\section{Relish signalling induces a starvation signature in pink1-mutant flies.}

Drosophila pink 1 mutants exhibit alterations in their circadian clock ${ }^{15}$, and here, we found that these flies also present defects in their gastrointestinal tract. Takeout is a Drosophila clock-controlled hormone that is primarily involved in feeding behaviour ${ }^{29}$ and is strongly expressed in the crop, which results in dilatation of the oesophagus that serves as a food reservoir (Fig. 6A), the fat body and the antennae of male flies. Takeout expression is also induced upon starvation ${ }^{29}$. We detected an increase in the level of Takeout in pink 1-mutant flies (Fig. 6B and Supplementary Table 2). Furthermore, the measurement of the transcript levels of this circadian output hormone revealed that this hormone was upregulated in pink1mutant flies and that this upregulation was partially suppressed in pink1, RelE20/+ double-mutant flies (Fig. 6C). The Drosophila genome encodes eight different insulin-like peptides (DILPs), and four of these peptides (DILP1, DILP2, DILP3 and DILP5) are functionally similar to human insulin and are produced by neuronal insulin-producing cells (IPCs) in the fly brain (Blue, Fig. 6A). Starvation, or low nutrient levels, decreases the release of DILPs by IPCs (reviewed in ${ }^{30}$ ). The increase in Takeout, a starvation marker, together with the report that another clock-regulated hormone, insulin-like peptide 2 (DILP2), is arrested in the cell bodies of IPCs in pink1-mutant flies ${ }^{16}$ led us to investigate whether these flies suffer from disruptions in metabolic and energy homeostasis. First, we focused on insulin, which is known to regulate dietary metabolism ${ }^{31}$. A confocal analysis of an epitope tagged DILP2 ${ }^{32}$ showed that pink 1 -mutant flies have higher levels of DILP2 in IPCs (Fig. 6D), which confirmed previous observations ${ }^{16}$. We then measured the total content of DILP2 peptide through an enzyme-linked immunosorbent assay (ELISA)based assay ${ }^{32}$ and found that the levels of DILP2 in aged pink1-mutant flies were lower than those in the controls (Fig. 6E). Systemic repression of insulin signalling leads to a FOXO-dependent activation of NFKB-Relish signalling, which results in the induction of triglyceride (TAG) metabolism upon starvation ${ }^{5}$.

Fasting induces the mobilization of stored TAGs from lipid droplets that are used by other tissues and organs as a source of energy. We subsequently measured the TAGs in control and pink1-mutant flies and found significant accumulation of TAGs in both young and aged pink1B9 flies (Fig. 6F), and Relish mutation restored the TAG levels in old flies to the normal levels (Fig. $6 \mathrm{H}$ ), in accordance with previous findings ${ }^{5,33}$. Taken together, these results link intestinal dysfunction in pink1-mutant flies to an imbalance in hormones related to feeding and changes in energy storage in adult flies.

\section{The suppression of intestinal toxicity in pink1-mutant flies is neuroprotective}

We previously showed that degeneration of the indirect flight muscles of pink1-mutant flies occurs in a non-cell autonomous manner through signalling between neurons and muscle cells ${ }^{11}$. Recent findings in models of PD have shown that a-synuclein pathology occurs via inter-organ communication between the 
gastrointestinal tract and the brain in mice (reviewed in ${ }^{34}$ ). Similar to those of mice, the central nervous system and gut of flies are interconnected. The Drosophila brain is connected to the midgut by neurons that produce insulin-like peptide 7 (ILP7) (Fig. 6A). The cell bodies of these neurons are located in the abdominal ganglion, and these cells innervate the midgut/hindgut junction and the rectal ampulla (reviewed in ${ }^{35}$ ).

To test whether gut-brain communication could cause neurotoxicity in pink1-mutant flies, we first suppressed Relish expression in the midgut by RNAi using the NP3084 midgut-specific ${ }^{36,37}$ Gal4 driver. This downregulation of Relish in the midgut decreased the overall levels of TAGs in pink1-mutant flies (Fig. 7A) and increased the level of fatty acid oxidation (Fig. 7B). These effects improved the mitochondrial function in the brains of pink1-mutant flies (Fig. 7C) and suppressed the inactivity defects (Fig. 7D) and the loss of DA neurons (Fig. 7E). We reason that the neurotoxic consequences of the activation of the immune response in pink1-mutant flies are linked to a gut-brain communication mechanism.

We subsequently tested whether the blockage of cell death in the intestine of pink 1-mutant flies was sufficient for the suppression of neurotoxicity. We targeted the expression of either Buffy, a Drosophila Bcl-2-like protein with anti-apoptotic activity ${ }^{38}$, or re-expressed Pink1 in the gut of pink1-mutant flies using the midgut driver. We found that the expression of either Buffy or Pink1 decreased the number of DCP1-positive cells (Figs. 8A and 8B) and prevented the loss of DA neurons in pink1-mutant flies (Fig. $8 \mathrm{C}$ ). We thus conclude that the suppression of intestinal dysfunction in pink 1 -mutant flies is sufficient for rescuing neurotoxicity.

\section{Discussion}

Mitochondria tune their performance to adjust their energy output to the requirements of individual cells. To achieve this tuning, mitochondria communicate with the cell nucleus using the retrograde response ${ }^{39}$, which modifies the flow of information in cells by altering the transcriptional control of cellular functions. Here, we found that Relish signalling serves as a retrograde response pathway that is activated by mitochondria and has neurotoxic consequences. Relish, the Drosophila orthologue of NF-kB in mammals, controls the expression of several immunity genes. Recent studies in humans and mice have shown that PINK1 plays a role in restraining innate immunity and that inflammation plays a positive role in PD 40. Here, we extend these observations and demonstrate that in flies, this inflammatory response is triggered in the intestine and communicated to the central nervous system. These conclusions build on the increasing body of evidence obtained from models of a-synuclein pathology (reviewed in ${ }^{34}$ ) that show a role for the gut-brain axis in the aetiology of PD.

We detected increased levels of enterocyte cell death in the intestines of pink1-mutant flies (Fig. 4). In Drosophila, Relish drives the removal of cells that are perceived unfit via apoptotic cell death ${ }^{41}$, and we reason that the release of DNA from defective mitochondria in pink1-mutant flies labels cells as unfit and 
prone to Relish-mediated apoptosis. It is also possible that a defective intestinal barrier in pink 1-mutant flies allows the invasion of gut bacteria in the intestine and activates an inflammatory response.

In Pink1-knockout mice, mitochondrial dysfunction induces an inflammatory response via the cGASSTING pathway that senses cytosolic DNA and acts upstream of the Relish orthologue NF-kB ${ }^{40}$. In Drosophila, dmSTING functions downstream of Relish to control viral infection ${ }^{42}$, and a previous study showed that its loss of function does not rescue the pink1-mutant phenotype ${ }^{43}$. Our data (Fig. 5) suggest that eya can act upstream of Relish to sense cytosolic DNA released by defective mitochondria in pink1mutant flies.

The defects in insulin signalling observed in pink1 mutants result from the abnormal trafficking of lipids between mitochondria and the endoplasmic reticulum, which in turn affects the formation of lipid vesicles and the release of neuropeptides such as DILPs ${ }^{16}$. These defects in lipid trafficking arise from the increased contacts between mitochondria and the endoplasmic reticulum observed in pink1-mutant flies ${ }^{12,16}$. Although partial suppression of Relish rescued the lipid defects in pink $1^{B 9}$ flies (Fig. $\left.6 \mathrm{H}\right)$, it did not rescue the release of DILP2 (Fig. 6G), confirming previous reports that insulin signalling acts upstream of Relish ${ }^{44}$.

Low levels of insulin are often associated with nutrient deficits and starvation, as indicated by an increased expression of Takeout (Figs. 6B and 6C) ${ }^{29}$, which results in reduced inhibition of FOXO by Akt and thus promotion of a stress response ${ }^{45}$. However, Relish can shape metabolic adaptation by attenuating Foxo-mediated lipolysis ${ }^{5}$, which leads to the accumulation of lipids ${ }^{46}$. Therefore, the sustained activation of Relish in pink1-mutant flies is likely to lead to the accumulation of lipids by blocking FOXO-mediated lipolysis.

We observed an accumulation of TAGs in pink1-mutant flies. Mitochondrial impairment has been shown to result in TAG accumulation ${ }^{47}$. We previously reported that dicarboxylate fatty acids are increased in pink 1 mutants, and this increase is likely linked to defects in fatty acid beta oxidation ${ }^{11}$. Therefore, the accumulation of TAGs in these mutants might result from the failure of fatty acid beta oxidation due to mitochondrial impairment.

In rodents, the gut-brain transmission of PD pathology by a-synuclein occurs via the vagus nerve in mice 34 . Both retrograde and anterograde vagal transport act to promote the bidirectional propagation of asynuclein toxicity to the brain and other organs in rats ${ }^{48}$.

Our results (Figs. 7 and 8) show that a gut-brain pathway is involved in neurotoxicity in a Drosophila model of PD. However, unlike the results obtained in a-synuclein models, our model showed that this pathway does not involve the transport of a toxic protein but rather a cell intrinsic signal induced by defective mitochondria. 
In Drosophila, neurons with cell bodies in the posterior segments of the abdominal ganglion of the ventral nerve cord (VNC) send axons that reach the posterior portion of the midgut (reviewed in ${ }^{35}$ ), which is the region where we detected intestinal dysfunction in pink 1-mutant flies. Because this link between the intestine and the CNS via the VNC is established by ILP7 neurons (Fig. 6A), these neurons, similar to the vagus nerve in rodents, might mediate toxicity between the midgut and brain in pink1-mutant flies. It would be interesting to test whether silencing the activity of these neurons would affect CNS toxicity in pink1-mutant flies.

Alternatively, the communication of toxicity from the intestine to the CNS in pink1 mutants could involve an indirect (humoural) mechanism. A previous study showed that AMPs induced by Relish directly induce mitochondrial depolarization ${ }^{49}$. AMPs in the intestine of pink1-mutant flies might act as a humoural signal to induce the loss of mitochondrial membrane potential in the CNS of these flies.

Our data add to an increasing body of evidence showing that signals from the gut can modulate brain activity. More specifically, our results suggest that mitochondrial toxicity in the gut can lead to adverse effects in the brain and that therapeutic interventions aiming to decrease gut toxicity might be a viable approach for restoring brain health in diseases caused by mitochondrial compromise.

\section{Abbreviations}

PD, Parkinson's Disease; PINK1, PTEN-induced kinase 1; mtDNA, mitochondria DNA; QC, quality control; Imd, immune deficiency; TAGs; triglycerides; CNS, central nervous system; AMPs, antimicrobial peptides; ISCs, intestinal stem cells; IPCs, insulin-producing cells; DAs, dopaminergic neurons

\section{Declarations}

\section{CONFLICT OF INTEREST}

The authors declare no conflicts of interest.

\section{ACKNOWLEDGEMENTS}

The proteomics analysis was performed at the Cambridge Centre for Proteomics by Renata Feret, Mike Deery and Yagnesh Umrania. We would like to thank the Vienna Drosophila RNAi Center, Bloomington Drosophila Stock Center, J. Cordero, A. Telemans, A. Franz and E. Rosato for providing the fly stocks, the Fly Facility, Department of Genetics, University of Cambridge, for maintaining the stocks, and T. Ashby and M. Patel for preparing the fly food. This work was funded by the UK Medical Research Council, intramural project MC_UU_00025/3 (RG94521).

\section{Methods}




\section{Genetics and Drosophila strains}

The fly stocks and crosses were maintained on standard cornmeal agar media at $25^{\circ} \mathrm{C}$. The strains used were the following: pink $1^{B 9}$, white $e^{1118}$ and $w$; elav>GAL4, which were previously described (Tufi et al., 2014), Canton-S backcrossed to $w^{1118}$ (Fedele et al., 2014), $w_{\text {;; }}$ Rel ${ }^{E 20}$ (BDSC_55714); $w^{\star} ;$ P $\{$ ppl-GAL4.P\}2 (BDSC_58768), $y w$; AttacinA::GFP (gift from A. Franz Lab), yw;; ILP2 HF (gift from A. Telemans), w; eya ${ }^{2}$ (BDSC_2285), w; UAS Relish RNAi (VDRC_P\{KK109851\}VIE-260B), $w^{*} ; P\{$ GawB $\} N P 3084$ (KSC_113094), w; UAS-Buffy, w; UAS-Pink-HA, THGal4, w; DILP2GAL4 (gift from E. Rosato), $y[1] w[*] P\{y[+t 7.7] w[+m C]=U A S-$ myrGFP.QUAS-mtdTomato-3xHA\}su(Hw)attP8; $P\{y[+t 7.7] w[+m C]=t r a n s-T a n g o\} a t t P 40$ (BDSC_77124) and w; ESG>GFP; TubGAL80 ${ }^{\text {ts }}$ (gift from J. Cordero).

To distinguish pink $1^{B 9}$ flies from flies with $\mathrm{X}$-nondisjunction, we crossed $\operatorname{pink} 7^{B 9}$ females to the Canton-S wild-type strain backcrossed to $w^{1118}$; thus, flies with nondisjunction have red eyes. This strategy was used for all crosses with mutants (i.e., $R e^{E 20}$ and $e y a^{2}$ ). For all other crosses, the flies were crossed to males carrying the FM7 balancer on the first chromosome, i.e., pink ${ }^{B 9}$;NP3084Gal4 x Fm7a; UAS-RelKK. All experiments involving adult flies were performed with males aged 3 to 5 days unless otherwise stated.

\section{Microarray acquisition and analysis}

RNA was obtained from the heads of male adult flies (six samples in total, three replicates of each genotype). The RNA quality was confirmed using an Agilent 2100 Bioanalyzer (Agilent Technologies, CA, USA). Detailed descriptions of the experimental protocols and raw data have been deposited in ArrayExpress under accession E-MTAB-6210. Differential expression was analysed using Partek Genomics Suite (Partek Inc., MO, USA) with an ANOVA model.

\section{Proteomics analysis}

Protein extracts from whole flies were prepared by grinding flies in radioimmunoprecipitation assay buffer (RIPA) (20 mM Tris pH 7.5, $150 \mathrm{mM} \mathrm{NaCl}, 1 \%(\mathrm{v} / \mathrm{v})$ Nonidet P40, 0.5\% (w/v) sodium deoxycholate, and $1 \mathrm{mM}$ EDTA) supplemented with $1 \mu \mathrm{g} / \mathrm{mL}$ leupeptin, $1 \mu \mathrm{g} / \mathrm{mL}$ antipain, $1 \mu \mathrm{g} / \mathrm{mL}$ chymostatin, 1 $\mu \mathrm{g} / \mathrm{mL}$ pepstatin and phosphatase inhibitor cocktail (PhosSTOP, Roche). The suspensions were cleared by centrifugation at $21,000 \mathrm{~g}$ and $4^{\circ} \mathrm{C}$ for 10 minutes, and the protein concentrations of the supernatants were measured using the Bradford assay (Bio-Rad). The cleared lysates were stored at $-80^{\circ} \mathrm{C}$ until proteomics analysis.

TMT labelling was performed according to the manufacturer's recommended protocol (https://www.thermofisher.com/order/catalogue/product/90110). One hundred micrograms of each digested protein sample was labelled individually with each of the 10 TMT tags. After labelling, the samples were combined, cleaned on a Sep-Pak C18 cartridge, dried and dissolved in $20 \mathrm{mM}$ ammonium 
formate ( $\mathrm{pH}$ 10). TMT peptide fractionation was performed using an Acquity ethylene-bridged hybrid $\mathrm{C} 18$ UPLC column (Waters; $2.1 \mathrm{~mm}$ i.d. x $150 \mathrm{~mm}$, particle size of $1.7 \mu \mathrm{m}$ ). Dried fractions were separated using the LC-MS/MS method as detailed below. The fractions were combined into pairs (i.e., the first fraction with the middle fraction) and analysed by LC-MS/MS using a Dionex Ultimate 3000 RSLC nanoUPLC (Thermo Fisher Scientific Inc, Waltham, MA, USA) system and a Lumos Orbitrap mass spectrometer (Thermo Fisher Scientific Inc, Waltham, MA, USA).

\section{Data analysis}

To explore the transcriptional changes in genes involved in the Drosophila immune response, transcripts with fold-changes (FCs) $\geq 2$ in pink1-mutant flies were cross-checked with a list of Drosophila genes potentially involved in the immune response, which was made available as a resource by the Lemaitre group (lemaitrelab.epfl.ch). The transcripts with FCs $\geq 2$ in pink1-mutant flies annotated to this list were then analysed using the iRegulon algorithm ${ }^{14}$ in Cytoscape (v3.5.1).

For proteomics analysis, the raw data files were processed using Proteome Discoverer v2.1 (Thermo Fisher Scientific) and Mascot (Matrix Science) v2.6. The data were aligned with the UniProt data from Pseudomonas aeruginosa (5584 sequences), which is the common repository of adventitious proteins (cRAP, version 1.0). All comparative analyses were performed with the $\mathrm{R}$ statistical language. The $\mathrm{R}$ package MSnbase ${ }^{51}$ was used for the processing of proteomics data. Briefly, this process entailed the removal of missing values (instances where a protein was identified but not quantified in all channels were rejected from further analysis), log2-transformation of the raw data, and subsequent sample normalization utilizing the 'diff.median' method in MSnbase (this translates all samples columns such that they all match the grand median). The differential abundances of the proteins were evaluated using the limma package, and the differences in protein abundances were statistically analysed using Student's $\mathrm{t}$-test with their variances moderated by the empirical Bayes method in limma. The $p$-values were adjusted for multiple testing using the Benjamini Hochberg method ${ }^{52}$.

\section{Locomotor assays}

Three- to four-day-old males were individually loaded into Drosophila Activity Monitors (DAM5) within $8 \times$ 65-mm glass Pyrex tubes (Trikinetics, Waltham, MA, USA) containing normal fly food. The flies were maintained at $25^{\circ} \mathrm{C}$ under a 12-hour light:12-hour dark (LD) cycle for at least 8 days. Sleep and activity data were analysed using the Sleep and Circadian Analysis MATLAB Program (SCAMP) developed by the Griffith lab ${ }^{53}$. The analyses were performed for 7 days starting at the first ZTO to allow acclimation. At least 16 flies of each genotype were used.

\section{Assay of the integrity of the intestinal barrier}


We measured the integrity/functionality of the intestinal barrier by detecting the presence of a nonabsorbable blue food dye outside the digestive tract after feeding ${ }^{54}$. The flies were transferred to food containing $2.5 \% \mathrm{w} / \mathrm{v}$ Erioglaucine (FD\&C Blue dye1-SIGMA) overnight ${ }^{55}$. Afterward, the flies were allowed to eat normal food for an additional 24 hours and then retransferred to dye-containing food for an additional 24 hours. The flies were scored after being fed normal food for 48 hours. Notably, in the analysis of Smurf flies fed normal food for 48 hours, a stained abdomen served as an indication of GI dysfunction, and we did not score different degrees of "smurfness". A pairwise test followed by a stack of $p$-values with an FDR of $10 \%$ was used to detect the significance of the differences among genotypes.

\section{Drug treatments}

Tacrolimus (FK-506 monohydrate, Sigma, F4679) was incorporated into the fly food to a final concentration of $5 \mathrm{mM}$. Flies treated with tacrolimus were transferred to drug-containing food up to 24 hours after hatching.

\section{Protein extraction and western blotting}

Ten to fifteen guts were dissected in PBS and maintained on ice until ready to be processed. Protein extraction was performed using NE-PER ${ }^{\text {TM }}$ Nuclear and Cytoplasmic Extraction Reagents (Thermo Scientific, 78833) following the manufacturer's instructions. The samples were separated using BioRad Precast gels and wet-blotted onto nitrocellulose.

\section{Antibodies and dyes}

The primary antibodies and dyes employed in this study were anti-a-tubulin (CST, 2125), anti-cleaved Drosophila Dcp-1 (CST, 9678), anti-HA (Roche Applied, 11583816001), anti-FLAG M2 (Sigma, F3165), anti-TH (Immunostar, 22941), anti-GFP (Abcam, ab13970), anti-Relish (RayBiotech, RB-14-0004-20), antids DNA (Abcam, ab27156), rhodamine phalloidin (Thermo Scientific, R415) and BODIPY 493/503 (Thermo Scientific, D3922). The secondary antibodies were goat anti-chicken IgY H\&L Alexa Fluor 488 (Abcam, ab 150173), goat anti-rabbit IgY H\&L Alexa Fluor 488 (Abcam, ab 150077), goat anti-mouse IgY H\&L Alexa Fluor 488 (Abcam, ab 150117), and goat anti-mouse IgG H\&L Alexa Fluor 555 (Abcam, ab150118).

\section{RNA extraction and quantitative real-time RT-PCR}

Total RNA was extracted from 10-15 freshly dissected guts using TRIzol (Ambion) and quantified by spectrophotometric analysis (Nanodrop, Thermo Scientific). Quantitative real-time PCR with reverse transcription (qRT-PCR) was performed with a real-time cycler (Applied Biosystems 7500 Fast Real-Time 
PCR Systems) using the SensiFAST SYBR Lo-ROX One-Step Kit (Bioline). Gene-specific primers were obtained from Sigma, and rp49 was used as a housekeeping gene.

\section{Immunofluorescence and confocal microscopy}

For brain imaging, the flies were fixed overnight at $4^{\circ} \mathrm{C}$ in $4 \% \mathrm{PFA} / 1 \%$ Triton/PBS. The brains were subsequently dissected in ice-cold PBS and blocked in 10\% normal goat serum PBS $/ 0.5 \%$ Triton overnight. Primary staining was performed at $4^{\circ} \mathrm{C}$ for 3 days, whereas the samples were incubated with the secondary antibodies for 2 hours at room temperature.

For intestinal imaging, the guts were dissected from live flies in PBS and fixed in 4\% PFA for 1 hour on ice. The staining protocol was identical to that used for the brain samples.

For DCP-1 staining, the guts were fixed in heptane-methanol fixative. The samples were mounted in Vectashield (Vector Laboratories), and fluorescence images were acquired with a Zeiss LSM880 confocal microscope.

\section{Microscopy-based assessment of mitochondrial function and morphology}

Measurements of the $\Delta \psi \mathrm{m}$ in fly brains were performed as previously described ${ }^{11}$. Briefly, fly brains were loaded for 40 minutes at room temperature with $40 \mathrm{nM}$ TMRM in loading buffer (10 mM HEPES pH 7.35, $156 \mathrm{mM} \mathrm{NaCl}, 3 \mathrm{mM} \mathrm{KCl}, 2 \mathrm{mM} \mathrm{MgSO}_{4}, 1.25 \mathrm{mM} \mathrm{KH}_{2} \mathrm{PO}_{4}, 2 \mathrm{mM} \mathrm{CaCl}_{2}$, and $10 \mathrm{mM}$ glucose), and the dye was present during the experiment. In these experiments, TMRM was used in the redistribution mode for the assessment of $\Delta \psi \mathrm{m}$, and therefore, a reduction in the TMRM fluorescence represents mitochondrial depolarization. Confocal images were obtained using a Zeiss LSM 880 confocal microscope equipped with a $40 x$ oil immersion objective. The illumination intensity was kept to a minimum $(0.1-0.2 \%$ of the laser output) to avoid phototoxicity, and the pinhole was set to yield an optical slice of $2 \mu \mathrm{m}$. The fluorescence was quantified by exciting TMRM using the $565-\mathrm{nm}$ laser and measured above $580 \mathrm{~nm}$. Zstacks of five $300-\mu \mathrm{m}^{2}$ fields were acquired from each brain, and the mean maximal fluorescence intensity of each group was measured.

\section{Analysis of dopaminergic neurons}

The brains from 25-day-old flies were dissected and stained with anti-tyrosine hydroxylase antibody (Immunostar) as previously described ${ }^{56}$. The brains were positioned in PBS $+0.1 \%$ Triton in a coverslip clamp chamber (ALA Scientific Instruments Inc., NY, USA) using a harp composed of platinum wire and nylon string and imaged by confocal microscopy. The numbers of tyrosine hydroxylase-positive neurons 
in the PPL1 cluster was determined for each brain hemisphere. The data acquired for the assessment of each genotype were obtained as a single experimental set prior to statistical analysis.

\section{Metabolic assays}

The assessment of DILP2 was performed by ELISA, whereas the levels of TAGs and beta oxidation were assessed through colorimetric assays using 96-well microtiter plates and an Infinite M200Pro multifunction reader (TECAN, Mannedorf, Switzerland).

For the metabolic assays, groups consisting of a maximum of 20 male flies were placed on normal foodcontaining vials, and the food was changed every 2 days until the desired age was reached, i.e., either 3 or 30 days, and maintained in an $12: 12 \mathrm{LD}$ at $25^{\circ} \mathrm{C}$. Day 0 was considered the day when the flies emerged from the pupal case. At the defined ageing stage, the flies were transferred into vials containing cotton soaked with water for 2 hours to minimize food contamination from ZT 2 to ZT 4. $\mathrm{CO}_{2}$-anaesthetized flies were then divided into groups of five flies and flash frozen for subsequent analysis. The TAG and glycogen assays were essentially performed as previously described ${ }^{57}$. Briefly, for TAG analysis, five adult flies were homogenized in $100 \mu \mathrm{L}$ of PBS $+0.05 \%$ Tween-20 (PBSTw) for 60 seconds on ice and immediately incubated at $70^{\circ} \mathrm{C}$ for 10 minutes for the inactivation of endogenous enzymatic activity. Forty microlitres of the fly samples and glycerol standards (SIGMA, G7793) were incubated together with either $40 \mu \mathrm{L}$ of PBST (for free glycerol measurements) or $40 \mu \mathrm{L}$ of TAG reagent (SIGMA T2449, for TAG measurements) at $37^{\circ} \mathrm{C}$ for 60 minutes.

After 3 minutes of centrifugation at full speed, $30 \mu \mathrm{l}$ of each sample (two technical replicates/biological sample) was transferred into a clear-bottom plate together with $100 \mu \mathrm{L}$ of free glycerol reagent (SIGMA, F6428) at $37^{\circ} \mathrm{C}$ for 5 minutes.

For insulin ELISA, the flies were maintained and aged as for the TAG assay with the modification that the flies were not starved for 2 hours but rather collected at ZT 2. The ELISA was performed as previously described ${ }^{32}$ with the modification that $5 \mu \mathrm{L}$ of anti-HA-HRP antibody diluted 1:350 in PBS was added to $50 \mu \mathrm{L}$ of protein samples as described by ${ }^{58}$. Subsequently, the TAG and DILP2 absorbances were divided by the protein concentration of the respective sample, which was measured by the Bradford assay. The results were normalized to the control group by dividing the average values ${ }^{59}$.

Similarly, for beta oxidation analysis, 10 flies of the desired age were collected and maintained on ice following the manufacturer's instructions.

For beta oxidation analysis, the flies were maintained and aged as described above with no starvation step and collected at ZT 2. Briefly, 15 flies (for each biological replicate) were anaesthetized, transferred to Eppendorf tubes, weighed and maintained on ice. Approximately $10 \mathrm{mg}$ of tissue was used for each replicate. The flies were subsequently homogenized in $200 \mu \mathrm{L}$ of provided lysis buffer, and the lysate was clarified by centrifugation at $4^{\circ} \mathrm{C}$. The Bradford assay was used for quantification of the protein 
concentration, and all the samples were normalized to $2 \mathrm{mg} / \mathrm{mL}$. The assay was then performed based on the protocol suggested by the manufacturer (BMR E-141). Incubation at $37^{\circ} \mathrm{C}$ was performed for 1 hour.

\section{Statistical analyses}

The statistical analyses were performed using GraphPad Prism 8 (www.graphpad.com). The data are presented as the mean values, and the error bars indicate the \pm SDs. In violin plots, the solid line represents the median, whereas the dotted lines represent the quartiles. The number of biological replicates per experimental variable $(n)$ is indicated in either the respective figure or the figure legend. The significance is indicated as follows: * indicates $p<0.05$, ** indicates $p<0.01$, *** indicates $p<0.001$, and $\star \star \star \star$ indicates $p<0.0001$.

\section{Digital image processing}

Fluorescence and western blot images were acquired as uncompressed bitmapped digital data (TIFF format) and processed using Adobe Photoshop with established scientific imaging workflows ${ }^{60}$. To visualize the pixel intensity, confocal images acquired with identical settings were processed using a 5tone heat map (f.64 Academy) in Photoshop.

\section{References}

1 Collins, L. V., Hajizadeh, S., Holme, E., Jonsson, I. M. \& Tarkowski, A. Endogenously oxidized mitochondrial DNA induces in vivo and in vitro inflammatory responses. J Leukoc Bio/ 75, 995-1000, doi:10.1189/jlb.0703328 (2004).

2 Celardo, I., Martins, L. M. \& Gandhi, S. Unravelling mitochondrial pathways to Parkinson's disease. British journal of pharmacology 171, 1943-1957, doi:10.1111/bph.12433 (2014).

3 Oka, T. et al. Mitochondrial DNA that escapes from autophagy causes inflammation and heart failure (vol 485, pg 251, 2012). Nature 490, 292-292, doi:10.1038/nature11515 (2012).

4 Myllymaki, H., Valanne, S. \& Ramet, M. The Drosophila imd signaling pathway. J Immuno/ 192, 3455-3462, doi:10.4049/jimmunol.1303309 (2014).

5 Molaei, M., Vandehoef, C. \& Karpac, J. NF-kappaB Shapes Metabolic Adaptation by Attenuating Foxo-Mediated Lipolysis in Drosophila. Dev Cell 49, 802-810 e806, doi:10.1016/j.devcel.2019.04.009 (2019).

6 Chinchore, Y., Gerber, G. F. \& Dolph, P. J. Alternative pathway of cell death in Drosophila mediated by NF-kappaB transcription factor Relish. Proc Natl Acad Sci U S A 109, E605-612, 
doi:10.1073/pnas.1110666109 (2012).

7 Kuraishi, T., Hori, A. \& Kurata, S. Host-microbe interactions in the gut of Drosophila melanogaster. Front Physio/ 4, 375, doi:10.3389/fphys.2013.00375 (2013).

8 Sheldon, B. C. \& Verhulst, S. Ecological immunology: Costly parasite defences and trade-offs in evolutionary ecology. Trends Ecol Evol 11, 317-321, doi:Doi 10.1016/0169-5347(96)10039-2 (1996).

9 Valtonen, T. M., Kleino, A., Ramet, M. \& Rantala, M. J. Starvation Reveals Maintenance Cost of Humoral Immunity. Evol Bio/ 37, 49-57, doi:10.1007/s11692-009-9078-3 (2010).

10 Liu, X. et al. Drosophila EYA regulates the immune response against DNA through an evolutionarily conserved threonine phosphatase motif. PLoS One 7, e42725, doi:10.1371/journal.pone.0042725 (2012).

11 Tufi, R. et al. Enhancing nucleotide metabolism protects against mitochondrial dysfunction and neurodegeneration in a PINK1 model of Parkinson's disease. Nat Cell Biol 16, 157-166, doi:10.1038/ncb2901 (2014).

12 Celardo, I. et al. Mitofusin-mediated ER stress triggers neurodegeneration in pink1/parkin models of Parkinson's disease. Cell Death Dis 7, e2271, doi:10.1038/cddis.2016.173 (2016).

13 Lemaitre, B. \& Hoffmann, J. The host defense of Drosophila melanogaster. Annu Rev Immuno/ 25, 697-743, doi:10.1146/annurev.immunol.25.022106.141615 (2007).

14 Janky, R. s. et al. iRegulon: from a gene list to a gene regulatory network using large motif and track collections. PLoS Comput Bio/ 10, e1003731 (2014).

15 Julienne, H., Buhl, E., Leslie, D. S. \& Hodge, J. J. L. Drosophila PINK1 and parkin loss-of-function mutants display a range of non-motor Parkinson's disease phenotypes. Neurobiology of Disease 104, 1523, doi:10.1016/j.nbd.2017.04.014 (2017).

16 Valadas, J. S. et al. ER Lipid Defects in Neuropeptidergic Neurons Impair Sleep Patterns in Parkinson's Disease. Neuron 98, 1155-1169 e1156, doi:10.1016/j.neuron.2018.05.022 (2018).

17 Park, J. et al. Mitochondrial dysfunction in Drosophila PINK1 mutants is complemented by parkin. Nature 441, 1157-1161 (2006).

18 Hedengren, M. et al. Relish, a central factor in the control of humoral but not cellular immunity in Drosophila. Mol Cell 4, 827-837 (1999).

19 Doktor, B., Damulewicz, M., Krzeptowski, W., Bednarczyk, B. \& Pyza, E. M. Effects of PINK1 mutation on synapses and behavior in the brain of Drosophila melanogaster. Acta Neurobiol Exp (Wars) 78, 231-241 (2018). 
$20 \mathrm{Li}$, Y. X. \& Dijkers, P. F. Specific calcineurin isoforms are involved in Drosophila toll immune signaling. J Immuno/ 194, 168-176, doi:10.4049/jimmunol.1401080 (2015).

21 Han, Z. S. \& Ip, Y. T. Interaction and specificity of Rel-related proteins in regulating Drosophila immunity gene expression. J Biol Chem 274, 21355-21361, doi:10.1074/jbc.274.30.21355 (1999).

22 Franz, A., Wood, W. \& Martin, P. Fat Body Cells Are Motile and Actively Migrate to Wounds to Drive Repair and Prevent Infection. Dev Cell 44, 460-470 e463, doi:10.1016/j.devcel.2018.01.026 (2018).

23 Rera, M., Clark, R. I. \& Walker, D. W. Intestinal barrier dysfunction links metabolic and inflammatory markers of aging to death in Drosophila. Proc Natl Acad Sci U S A 109, 21528-21533, doi:10.1073/pnas.1215849110 (2012).

24 Naszai, M., Carroll, L. R. \& Cordero, J. B. Intestinal stem cell proliferation and epithelial homeostasis in the adult Drosophila midgut. Insect Biochem Mol Biol 67, 9-14, doi:10.1016/j.ibmb.2015.05.016 (2015).

25 Micchelli, C. A. \& Perrimon, N. Evidence that stem cells reside in the adult Drosophila midgut epithelium. Nature 439, 475-479, doi:10.1038/nature04371 (2006).

26 Harsh, S., Heryanto, C. \& Eleftherianos, I. Intestinal lipid droplets as novel mediators of hostpathogen interaction in Drosophila. Biol Open, doi:10.1242/bio.039040 (2019).

27 West, A. P. et al. Mitochondrial DNA stress primes the antiviral innate immune response. Nature 520, 553-557, doi:10.1038/nature14156 (2015).

28 Shokri, L. et al. A Comprehensive Drosophila melanogaster Transcription Factor Interactome. Cell Rep 27, 955-970 e957, doi:10.1016/j.celrep.2019.03.071 (2019).

29 Sarov-Blat, L., So, W. V., Liu, L. \& Rosbash, M. The Drosophila takeout gene is a novel molecular link between circadian rhythms and feeding behavior. Cell 101, 647-656, doi:Doi 10.1016/S00928674(00)80876-4 (2000).

30 Ahmad, M., He, L. \& Perrimon, N. Regulation of insulin and adipokinetic hormone/glucagon production in flies. Wiley Interdiscip Rev Dev Biol, e360, doi:10.1002/wdev.360 (2019).

31 Post, S. et al. Drosophila Insulin-Like Peptides DILP2 and DILP5 Differentially Stimulate Cell Signaling and Glycogen Phosphorylase to Regulate Longevity. Front Endocrinol (Lausanne) 9, 245, doi:10.3389/fendo.2018.00245 (2018).

32 Park, S. et al. A genetic strategy to measure circulating Drosophila insulin reveals genes regulating insulin production and secretion. PLoS Genet 10, e1004555, doi:10.1371/journal.pgen.1004555 (2014). 
33 Heller, S. et al. Intestinal inflammation requires FOXO3 and prostaglandin E2-dependent lipogenesis and elevated lipid droplets. Am J Physiol Gastrointest Liver Physio/ 310, G844-854, doi:10.1152/ajpgi.00407.2015 (2016).

$34 \mathrm{Kim}, \mathrm{S}$. et al. Transneuronal Propagation of Pathologic alpha-Synuclein from the Gut to the Brain Models Parkinson's Disease. Neuron 103, 627-641 e627, doi:10.1016/j.neuron.2019.05.035 (2019).

35 Miguel-Aliaga, I., Jasper, H. \& Lemaitre, B. Anatomy and Physiology of the Digestive Tract of Drosophila melanogaster. Genetics 210, 357-396, doi:10.1534/genetics.118.300224 (2018).

36 Yin, S., Qin, Q. H. \& Zhou, B. Functional studies of Drosophila zinc transporters reveal the mechanism for zinc excretion in Malpighian tubules. Bmc Bio/ 15, doi:ARTN 12 10.1186/s12915-0170355-9 (2017).

37 Nehme, N. T. et al. A model of bacterial intestinal infections in Drosophila melanogaster. PLoS Pathog 3, 1694-1709, doi:ARTN e17310.1371/journal.ppat.0030173 (2007).

38 Quinn, L. et al. Buffy, a Drosophila Bcl-2 protein, has anti-apoptotic and cell cycle inhibitory functions. $E M B O$ J 22, 3568-3579, doi:10.1093/emboj/cdg355 (2003).

39 Allen, J. F. Control of gene expression by redox potential and the requirement for chloroplast and mitochondrial genomes. J Theor Biol 165, 609-631, doi:10.1006/jtbi.1993.1210 (1993).

40 Sliter, D. A. et al. Parkin and PINK1 mitigate STING-induced inflammation. Nature 561, 258-262, doi:10.1038/s41586-018-0448-9 (2018).

41 Meyer, S. N. et al. An ancient defense system eliminates unfit cells from developing tissues during cell competition. Science 346, 1258236, doi:10.1126/science.1258236 (2014).

42 Liu, Y. et al. Inflammation-Induced, STING-Dependent Autophagy Restricts Zika Virus Infection in the Drosophila Brain. Cell Host Microbe 24, 57-68 e53, doi:10.1016/j.chom.2018.05.022 (2018).

43 Lee, J. J., Andreazza, S. \& Whitworth, A. J. The STING pathway does not contribute to behavioural or mitochondrial phenotypes in Drosophila Pink1/parkin or mtDNA mutator models. Sci Rep 10, 2693, doi:10.1038/s41598-020-59647-3 (2020).

44 Karpac, J., Younger, A. \& Jasper, H. Dynamic coordination of innate immune signaling and insulin signaling regulates systemic responses to localized DNA damage. Dev Cell 20, 841-854, doi:10.1016/j.devcel.2011.05.011 (2011).

45 Puig, O., Marr, M. T., Ruhf, M. L. \& Tjian, R. Control of cell number by Drosophila FOXO: downstream and feedback regulation of the insulin receptor pathway. Genes Dev 17, 2006-2020, doi:10.1101/gad.1098703 (2003). 
46 Rynes, J. et al. Activating transcription factor 3 regulates immune and metabolic homeostasis. Mol Cell Bio/ 32, 3949-3962, doi:10.1128/MCB.00429-12 (2012).

47 Song, W. et al. Activin signaling mediates muscle-to-adipose communication in a mitochondria dysfunction-associated obesity model. Proc Natl Acad Sci U S A, doi:10.1073/pnas.1708037114 (2017).

48 Van Den Berge, N. et al. Evidence for bidirectional and trans-synaptic parasympathetic and sympathetic propagation of alpha-synuclein in rats. Acta Neuropatho/ 138, 535-550, doi:10.1007/s00401019-02040-w (2019).

49 Badinloo, M. et al. Overexpression of antimicrobial peptides contributes to aging through cytotoxic effects in Drosophila tissues. Arch Insect Biochem Physio/ 98, e21464, doi:10.1002/arch.21464 (2018).

50 Nassel, D. R., Kubrak, O. I., Liu, Y. T., Luo, J. N. \& Lushchak, O. V. Factors that regulate insulin producing cells and their output in Drosophila. Front Physio/ 4, doi:ARTN 252 10.3389/fphys.2013.00252 (2013).

51 Gatto, L. \& Lilley, K. S. MSnbase-an R/Bioconductor package for isobaric tagged mass spectrometry data visualization, processing and quantitation. Bioinformatics 28, 288-289, doi:10.1093/bioinformatics/btr645 (2012).

52 Benjamini, Y. \& Hochberg, Y. Controlling the False Discovery Rate: A Practical and Powerful Approach to Multiple Testing. Journal of the Royal Statistical Society 57, 289-300 (1995).

53 Donelson, N. C. et al. High-resolution positional tracking for long-term analysis of Drosophila sleep and locomotion using the "tracker" program. PLoS One 7, e37250, doi:10.1371/journal.pone.0037250 (2012).

54 Rera, M. et al. Modulation of longevity and tissue homeostasis by the Drosophila PGC-1 homolog. Cell Metab 14, 623-634, doi:10.1016/j.cmet.2011.09.013 (2011).

55 Martins, R. R., McCracken, A. W., Simons, M. J. P., Henriques, C. M. \& Rera, M. How to Catch a Smurf? - Ageing and Beyond... In vivo Assessment of Intestinal Permeability in Multiple Model Organisms. Bio Protoc 8, doi:10.21769/BioProtoc.2722 (2018).

56 Whitworth, A. J. et al. Increased glutathione S-transferase activity rescues dopaminergic neuron loss in a Drosophila model of Parkinson's disease. Proc Natl Acad Sci U S A 102, 8024-8029 (2005).

57 Tennessen, J. M., Barry, W. E., Cox, J. \& Thummel, C. S. Methods for studying metabolism in Drosophila. Methods 68, 105-115, doi:10.1016/j.ymeth.2014.02.034 (2014).

58 Moraru, A. et al. Elevated Levels of the Reactive Metabolite Methylglyoxal Recapitulate Progression of Type 2 Diabetes. Cell Metab 27, 926-934 e928, doi:10.1016/j.cmet.2018.02.003 (2018). 
60 Wexler, E. J. (Lynda.com, Inc., Ventura, 2008).

\section{Figures}

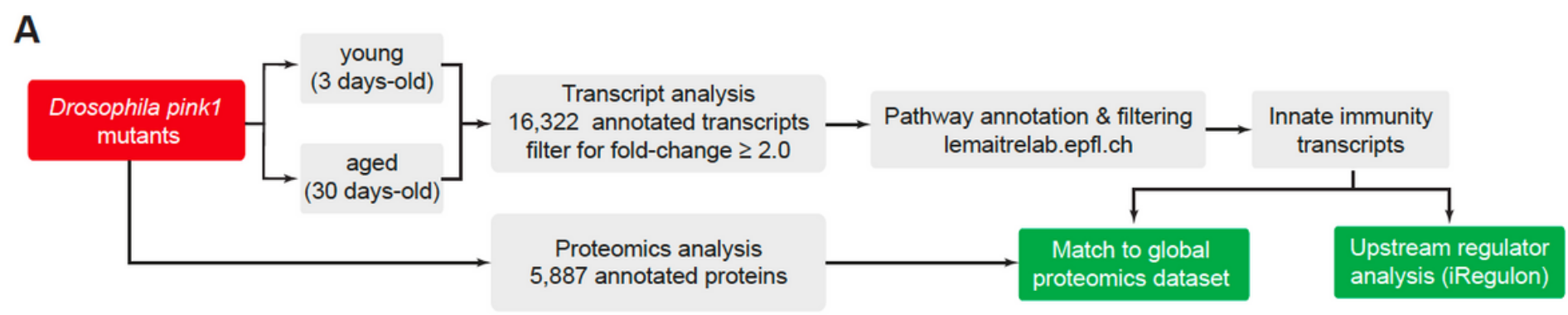

B

\begin{tabular}{|c|c|c|c|c|c|c|}
\hline & \multirow[b]{2}{*}{ Name } & \multicolumn{4}{|c|}{ mRNA } & \multirow{2}{*}{$\begin{array}{l}\text { Relish } \\
\text { Target? }\end{array}$} \\
\hline & & Symbol & Young & Aged & Protein & \\
\hline \multirow{4}{*}{1} & Sensory neuron membrane protein I & Snmp1 & $\infty$ & 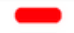 & ND & \\
\hline & PGRP-SC1b & PGRP-SC1b & $\longrightarrow$ & $\square$ & ND & $\varpi$ \\
\hline & PGRP-SD & PGRP-SD & $\rightarrow$ & $\infty$ & $\infty$ & $\varpi$ \\
\hline & Scavenger receptor class C, type II & $\mathrm{Sr}-\mathrm{Cll}$ & $\longrightarrow$ & & ND & \\
\hline II & Nimrod C1 & NimC1 & 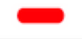 & 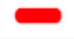 & 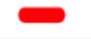 & \\
\hline \multirow{2}{*}{ III } & lectin-24A & lectin-24A & 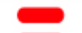 & $\square$ & ND & $\varpi$ \\
\hline & lectin-37Da & lectin-37Da & & 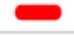 & ND & $\varpi$ \\
\hline \multirow{3}{*}{ V } & MstProx & MstProx & 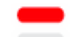 & 0 & ND & $\varpi$ \\
\hline & unpaired 3 & upd3 & 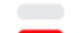 & $\longrightarrow$ & ND & $\varpi$ \\
\hline & Diedel & Diedel & $\longrightarrow$ & 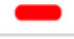 & 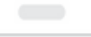 & \\
\hline \multirow{2}{*}{ VI } & prophenol oxidase A1 & proPO-A1 & 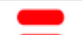 & $\square$ & $N D$ & \\
\hline & prophenoloxidase 45 & proPO45 & & 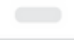 & ND & \\
\hline VII & CG5550 & CG5550 & $\infty$ & $\infty$ & 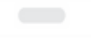 & $\varpi$ \\
\hline \multirow{8}{*}{ IX } & Lysozyme X & LysX & $\square$ & $\infty$ & 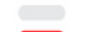 & $\varpi$ \\
\hline & Attacin-A & AttA & 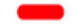 & & $\hookrightarrow$ & $\varpi$ \\
\hline & Drosomycin & Drs & 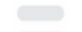 & $\infty$ & $\infty$ & $\varpi$ \\
\hline & Drosomycin-like 3 & Drsl3 & $\longrightarrow$ & 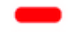 & $N D$ & \\
\hline & Drosomycin-like 4 & Drsl4 & & & $\infty$ & \\
\hline & Drosomycin-like 5 & Drsl5 & & $\rightarrow$ & $\infty$ & \\
\hline & Metchnikowin & Mtk & 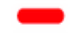 & 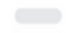 & ND & $\varpi$ \\
\hline & Listericin & Listericin & 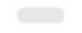 & $\infty$ & ND & $\varpi$ \\
\hline
\end{tabular}

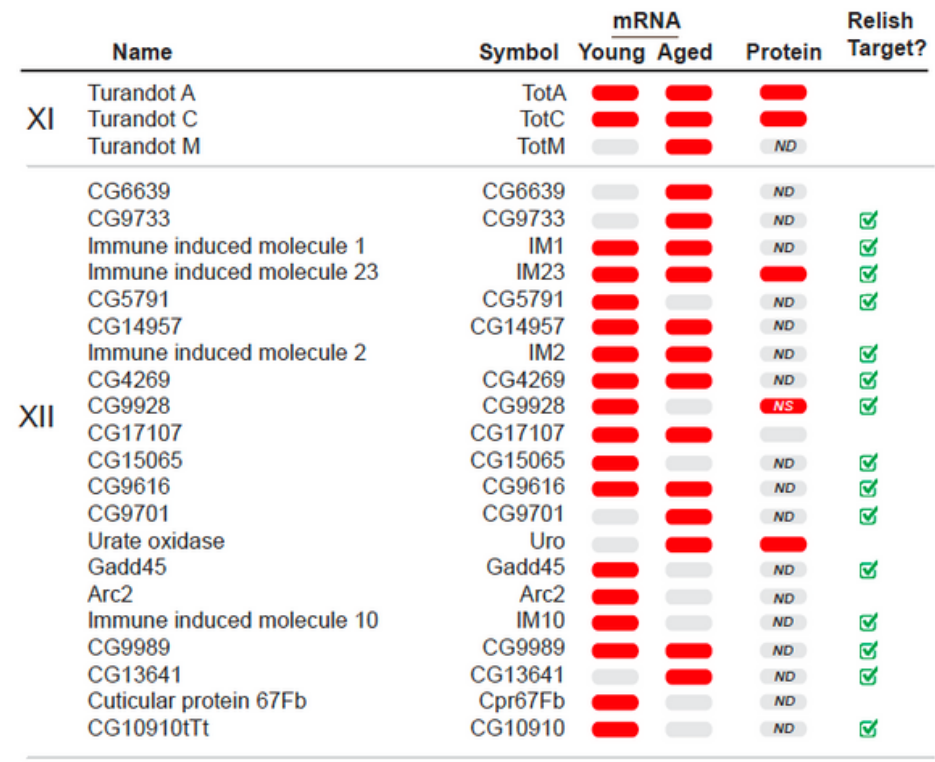

Legend $\square$ Fold-change $\geq 2$

I - Family of receptors potentially involved in microbial recognition II - Phagocytosis III - Encapsulation V-Signaling $2 \geq$ Fold-change $\geq-2$

\section{Figure 1}

In silico identification of Relish as a regulator of transcriptional changes in pink1-mutant flies. (A) Workflow used for the characterization of transcripts and proteins involved in the immune response in Drosophila pink1 mutants. Transcripts upregulated by more than two-fold were matched to a list of all genes potentially involved in responses to microbial infection, which are available from the Lemaitre group (lemaitrelab.epfl.ch). After annotation, these genes were matched to the corresponding proteins through global quantitative proteomics analysis. The transcripts were also matched to upstream regulators using the iRegulon algorithm in Cytoscape. (B) Relish targets in pink1-mutant flies. Transcripts that are potentially involved in the Drosophila immune response, as curated by the Lemaitre group, are shown. The iRegulon algorithm predicted that the majority (60\%) of the annotated transcripts are 
regulated by Relish. The detected proteins matched to each of the filtered transcripts are also shown. Transcripts and proteins upregulated by at least two-fold are shown in red colour. ND, not detected; NS, not significant. This figure is related to Supplementary Tables 1, 2 and 3.

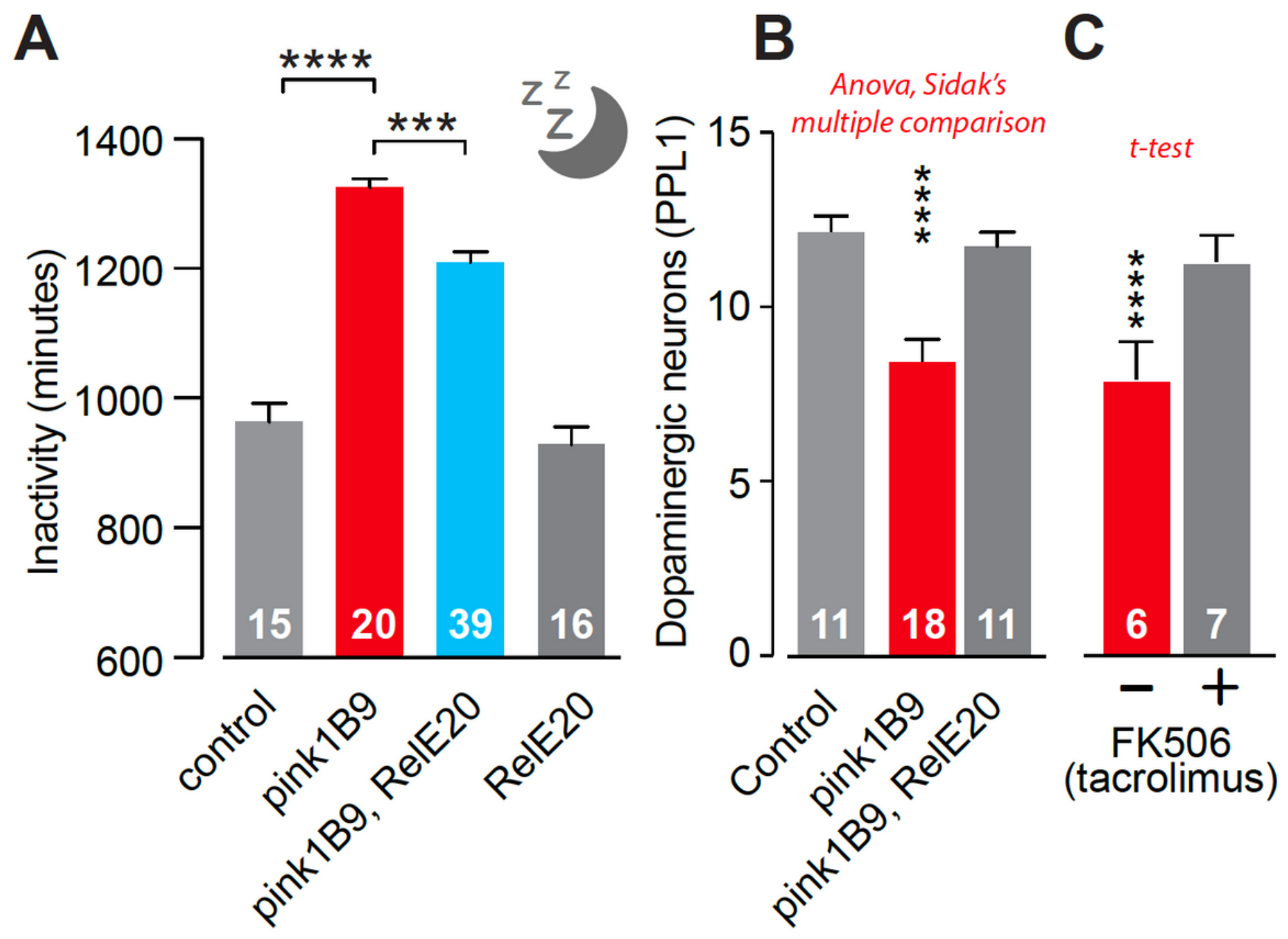

Figure 2

Neuronal defects in pink1-mutant flies are rescued by genetic and pharmacological inhibition of the immune response. (A) Sleep defects in pink1 mutants are suppressed by a Relish mutation (asterisks, one-way ANOVA with Tukey's multiple comparison test). (B) Relish mutation prevents loss of the PPL1 cluster of dopaminergic neurons in pink1-mutant flies (means \pm SDs; asterisks, one-way ANOVA with the Sidak multiple comparison test). (C) Dietary supplementation with tacrolimus (5 $\mathrm{mM}$ ) rescues the loss of dopaminergic neurons in the PPL1 cluster in pink1-mutant flies (means \pm SDs; asterisks, two-tailed Student's t-test). The genotypes in (A) and (B) are the following: control: w; CS/+, pink1B9: pink1B9; +; +, pink1B9, RelE20: pink1B9; +; RelE20/+, RelE20: w; +; RelE20/+. The genotype in (C) is pink1B9; +; +. 
A
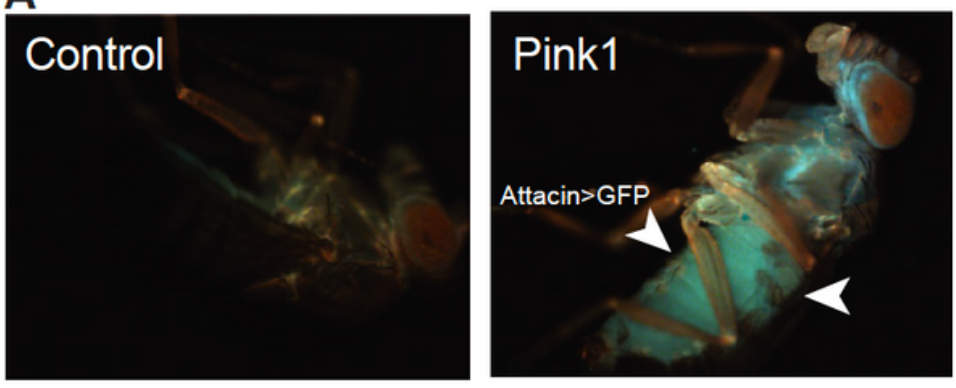

C

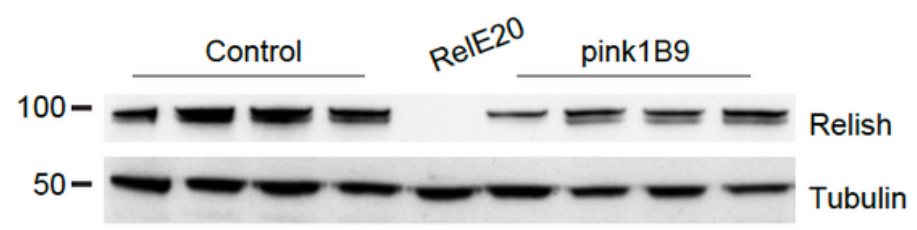

$\mathbf{E}$

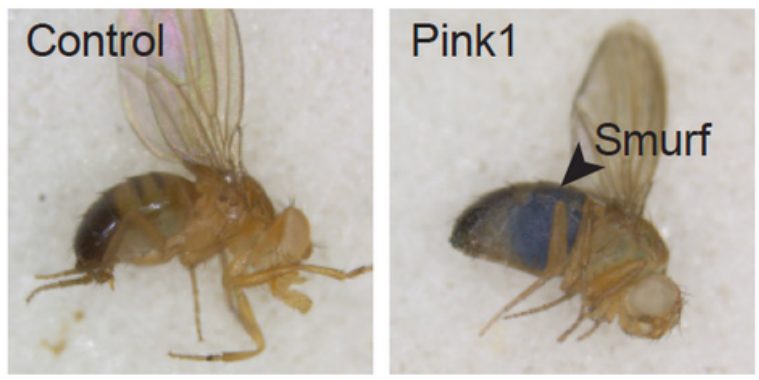

B
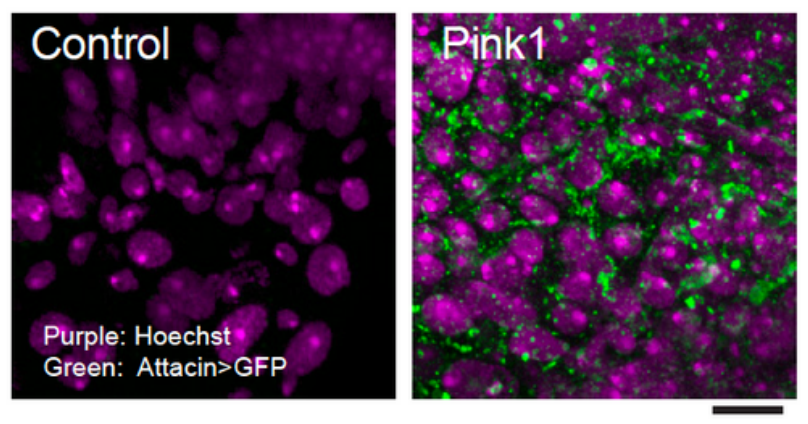

$10 \mu \mathrm{m}$

D
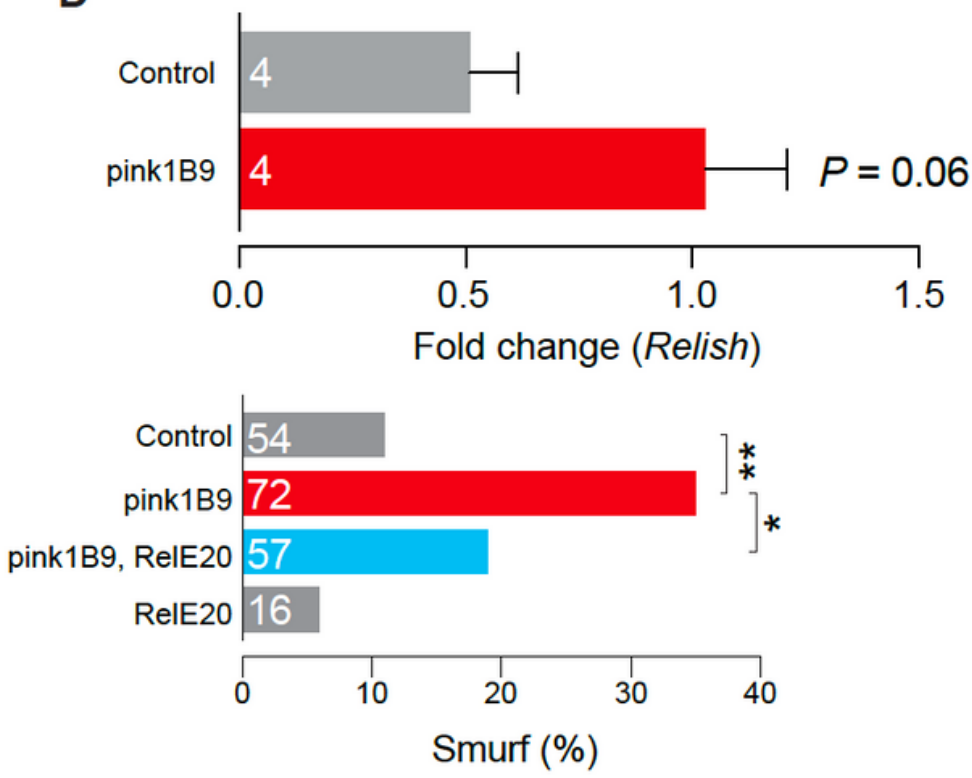

\section{Figure 3}

pink1-mutant flies show intestinal dysfunction. (A) Representative images showing increased expression of an Attacin-GFP reporter in pink1-mutant flies, whereas no detectable expression was observed in agematched control flies. (B) Analysis of gut enterocytes in flies expressing the Attacin-GFP reporter. Representative images are shown. (C) The levels of cytosolic Relish in the intestine are decreased in pink1-mutant flies. Lysates were probed with the indicated antibodies. Lysates from Relish-mutant flies were included to confirm the specificity of the anti-Relish antibody. Four biological replicates of the control and pink1-mutant flies were included in the analysis. (D) The Relish transcript levels are increased in pink1 mutants (means \pm SEMs; $P$ - value, two-tailed Student's t-test). (E) pink1 mutants exhibit a loss of the integrity of their intestinal barrier. The analysis was performed using the Smurf assay. Left: representative images showing a Smurf-positive pink1-mutant fly (arrowhead). Right: quantification of Smurf-positive flies. The defects in the intestinal barrier observed in pink1-mutant flies are reduced by the Relish mutation. The numbers in the bars represent the number of flies tested. Chi-Square with Analysis Stack of $p$-values, FDR $=10 \%$. The genotypes in $(A)$ are the following: control: $y w ; A t t a c i n A:: G F P, ~ P i n k 1$ : pink1B9; AttacinA::GFP. The genotypes in (C) to (E) are the following: control: w; CS/+, pink1B9: pink1B9; +; +, pink1B9, RelE20: pink1B9; +; RelE20/+, RelE20: w; +; RelE20/+. 


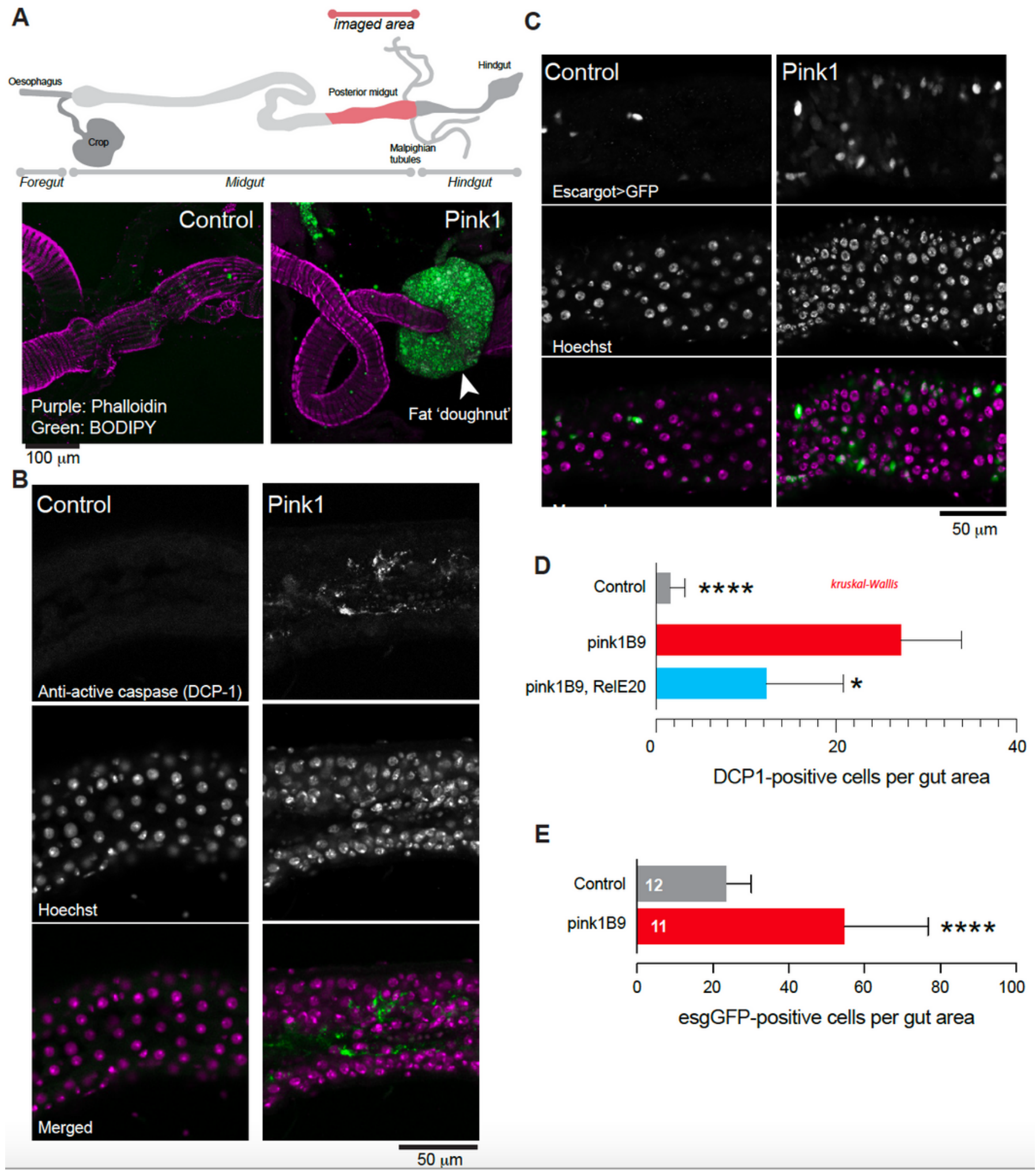

\section{Figure 4}

Intestinal dysfunction in pink1-mutant flies is rescued by a Relish mutation. (A) Analysis of the gastrointestinal tract showed a ring of lipids (fat 'doughnut') around the posterior midgut of pink1-mutant flies (arrowhead). Purple, phalloidin; Green, BODIPY. An illustration of the imaged region of the gut is shown. (B and D) Caspase activation in the intestine of pink1-mutant flies is blocked by a Relish mutation. Purple, cell nuclei; Green, Dcp-1. The quantitation of Dcp-1-positive cells in the posterior midgut 
shows that a Relish mutation decreases apoptosis of the enterocytes of pink1-mutant flies (means \pm SDs; asterisks, one-way ANOVA with the Kruskal-Wallis multiple comparison test). (C and E) Increased Escargot expression in the enterocytes of pink1-mutant flies. The gut areas analysed are 425 micron posterior midgut region, just anterior to the Malpighian tubules/hindgut junction. The genotypes in (A), (B) and (D) are the following: control: w; CS/+, pink1B9: pink1B9; +; +, pink1B9, RelE20: pink1B9; +; RelE20/+, RelE20: w; +; RelE20/+. The genotypes in (C) and (E) are the following: control: w; Esg>GFP; +, Pink1: pink1B9, Esg>GFP;+.

A
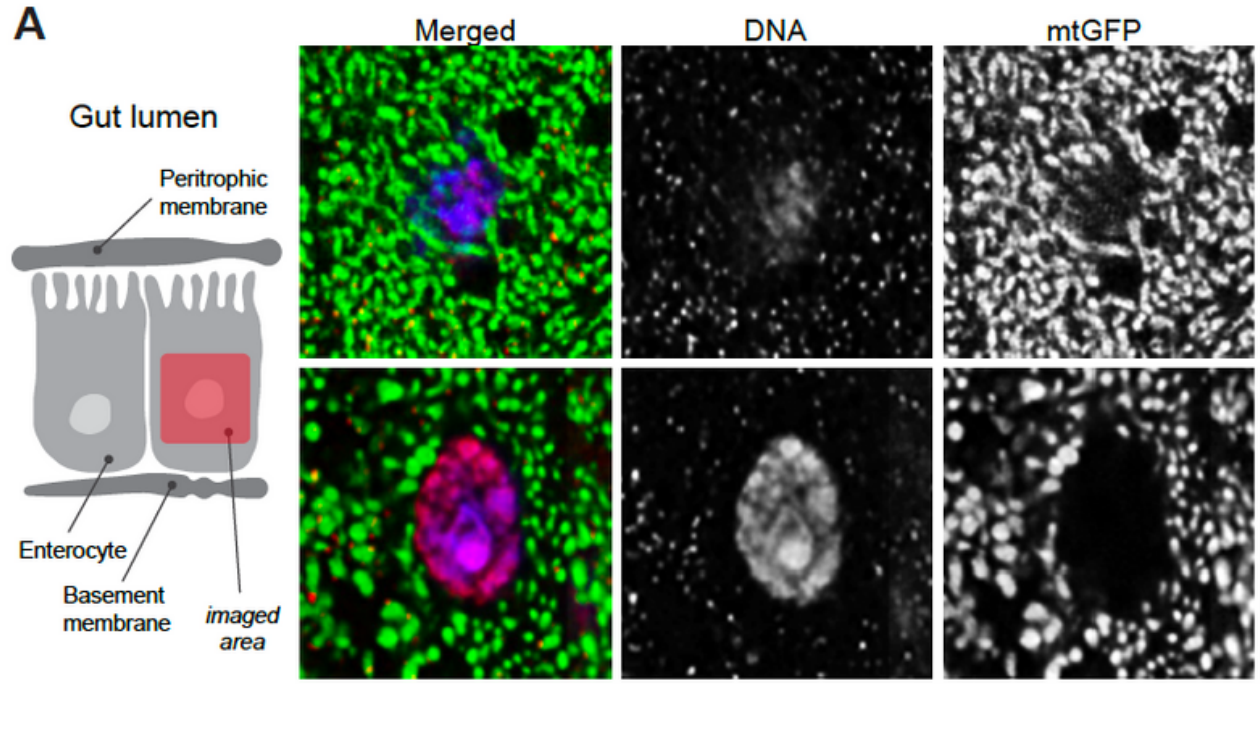

C
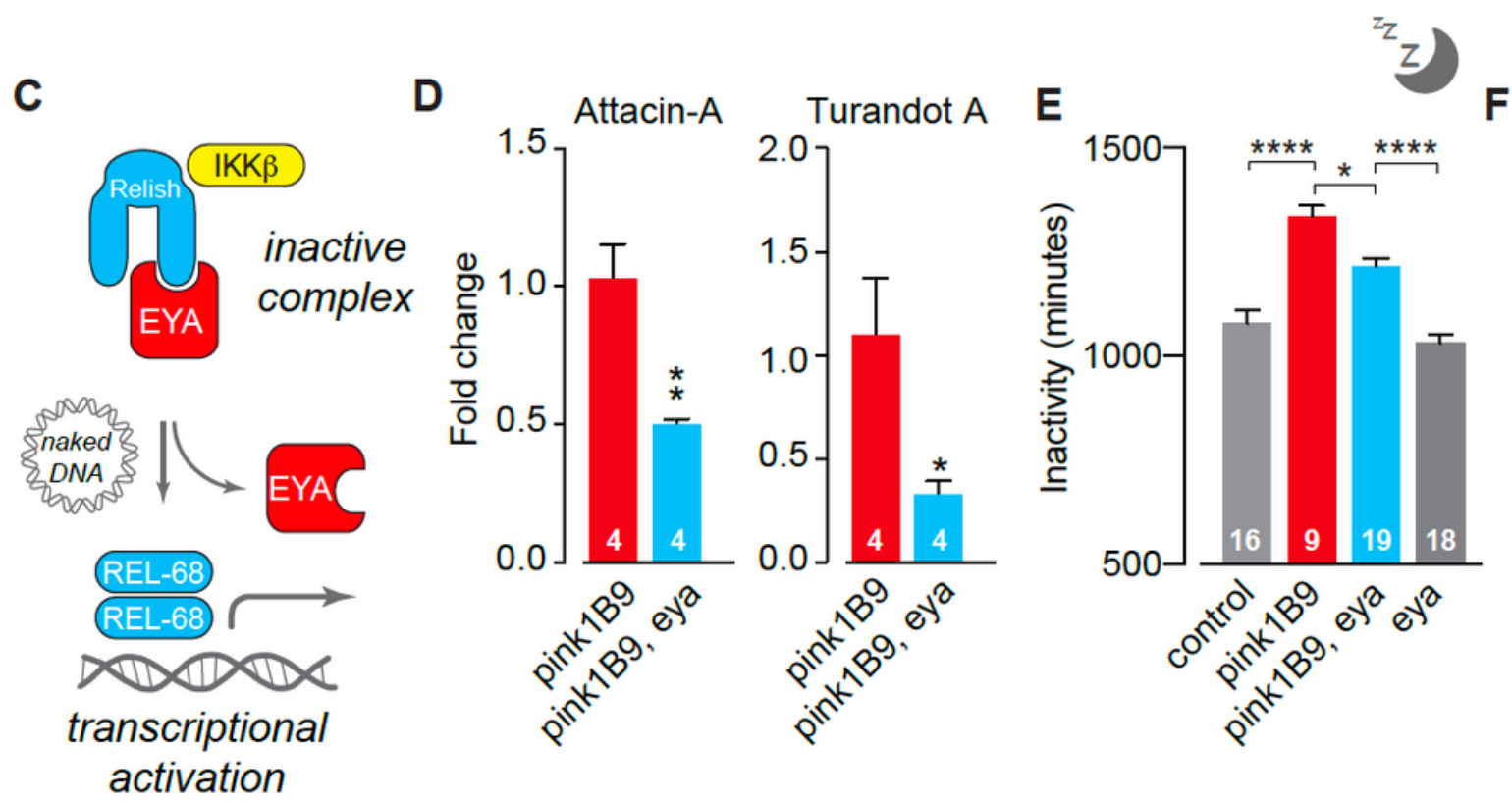

$\mathbf{F}$

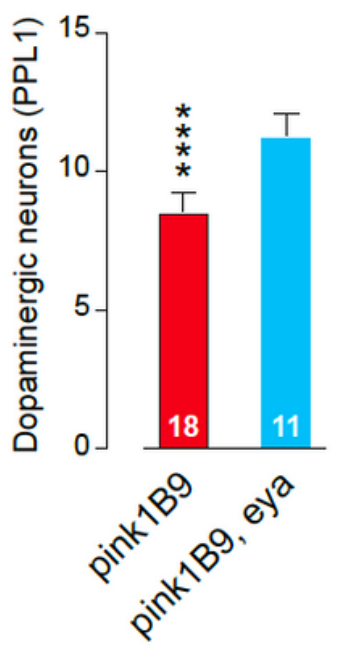

\section{Figure 5}

Mutation in a sensor of the innate immune response to DNA rescues the intestinal dysfunction of pink1mutant flies. (A-B) Decrease in cytosolic DNA colocalizing with mitochondria in pink1-mutant flies. Enterocytes expressing mtGFP were stained with an anti-DNA antibody and Hoechst to visualize the cell nucleus. The colocalization of DNA with mitochondria was assessed using Pearson's correlation coefficients from five regions of interest (ROIs, 10 microns) in each gut (asterisks, two-tailed Student's t- 
test). (C) Illustration of the proposed model 10 of the Eya-dependent activation of Relish. Eya associates with IKK $\beta$ and Relish in the cytoplasm. The immune response to undigested DNA in flies requires the eya gene. (D) The eya2 mutation reduces the expression of the innate immunity transcripts Attacin-A and Turandot $A$ in the intestines of pink1-mutant flies (means \pm SEMs; asterisks, two-tailed Student's t-test). (E and $F$ ) The eya2 mutation rescues sleep defects $(E)$ and the loss of dopaminergic neurons in the PPL1 cluster (F) in pink1-mutant flies (means \pm SDs; asterisks, one-way ANOVA with Tukey's multiple comparison test $(E)$, two-tailed Student's t-test $(F)$ ). The genotypes in $(A)$ and $(B)$ are the following: control: w; np3084Gal4/+; UASmitoGFP/+, Pink1: pink1B9, np3084Gal4/+; UASmitoGFP/+. The genotypes in (C) to $(F)$ are the following: control: w; CS/+, pink1B9: pink1B9; +; +, pink1B9, eya: pink1B9; eya2/+; +, eya: w; eya2/+; +.

A

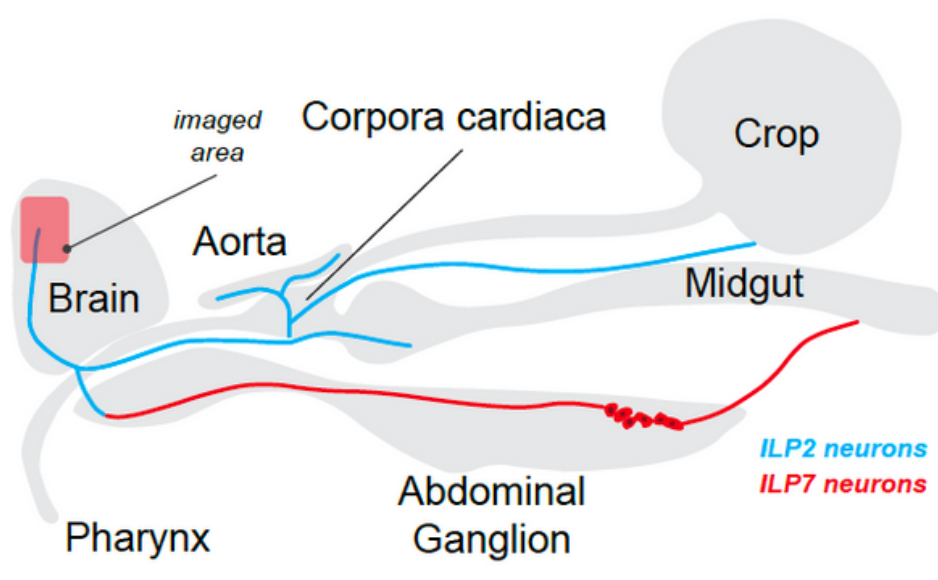

B

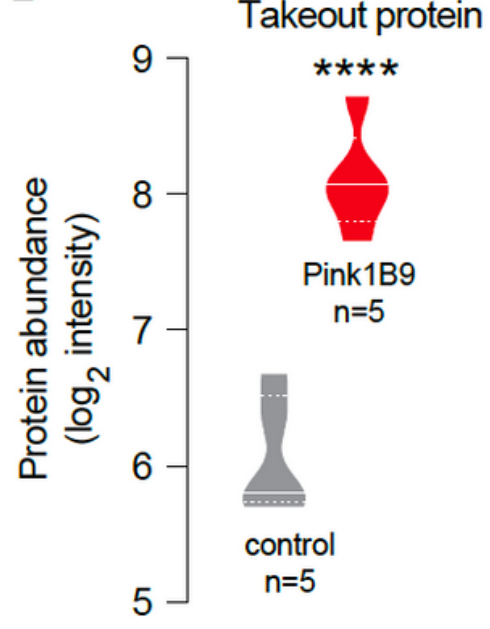

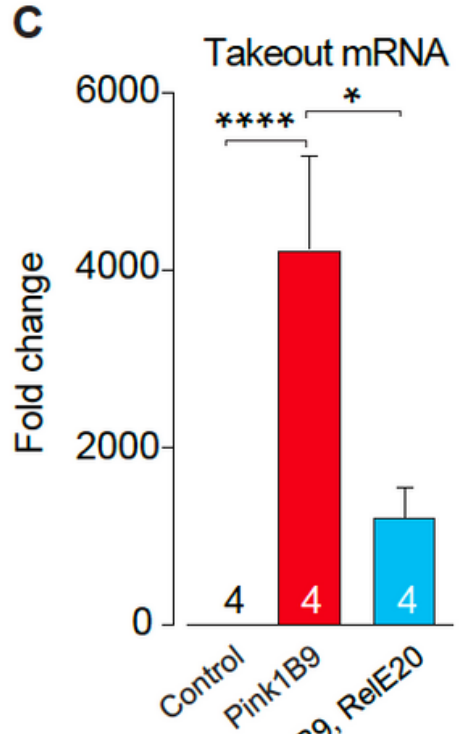

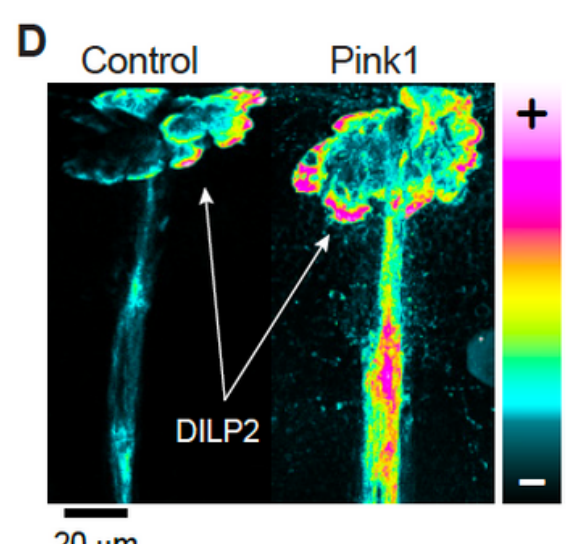

$20 \mu \mathrm{m}$
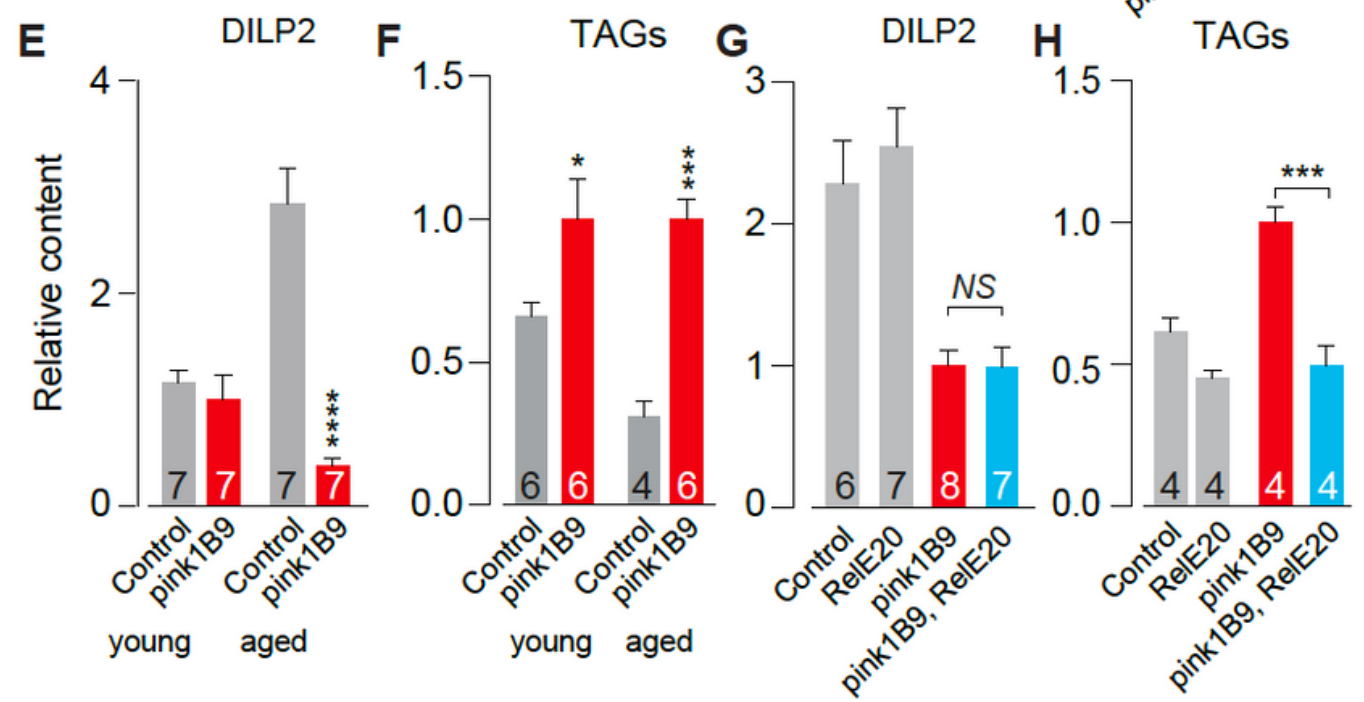

\section{Figure 6}

pink1-mutant flies show increases in metabolic markers of starvation. (A) Insulin-producing neurons (IPCs, blue) are wired between the Drosophila nervous system and gut (adapted from 50). (B) Increased levels of Takeout in pink1-mutant flies. The protein levels were measured by mass spectrometry 
(asterisks, corrected p-value using the Benjamini Hochberg method). This figure is related to Supplementary Table 2. (C) The upregulation of Takeout in aged (30-day-old) pink1-mutant flies is partially reversed by a Relish mutation (asterisks, one-way ANOVA with Dunnett's multiple comparison test). (D) Analysis of IPCs expressing an epitope-tagged DILP2. Higher levels of DILP2 are detected in the IPCs of pink1-mutant flies. The intensity levels are visualized using a 5-tone heat map. (E) Decreased levels of circulating DILP2 in aged pink1-mutant flies (means \pm SEMs; asterisks, two-way ANOVA with Tukey's multiple comparison test). (F) Increased levels of circulating triglycerides in pink1-mutant flies (means \pm SEMs; asterisks, one-way ANOVA with Tukey's multiple comparison test). (G) Relish mutation does not affect the loss of circulating DILP2 in pink1-mutant flies (means \pm SEMs; asterisks, one-way ANOVA with Tukey's multiple comparison test). (H) Relish mutation blocks the age-dependent increase in circulating triglycerides in pink1-mutant flies (means \pm SEMs; asterisks, one-way ANOVA with Tukey's multiple comparison test). The genotypes in (B) to $(C)$ and $(E)$ to $(H)$ are the following: control: $w ; C S /+$, pink1B9: pink1B9; +; +, pink1B9, RelE20: pink1B9; +; RelE20/+, RelE20: w; +; RelE20/+. The genotypes in (D) are the following: control: w;+; llp2/+, Pink1B9: pink1B9;+; Ilp2/+. 
A

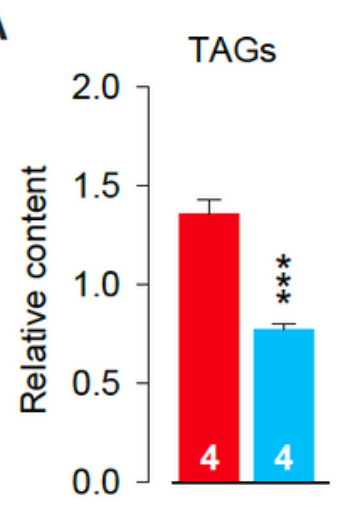

B

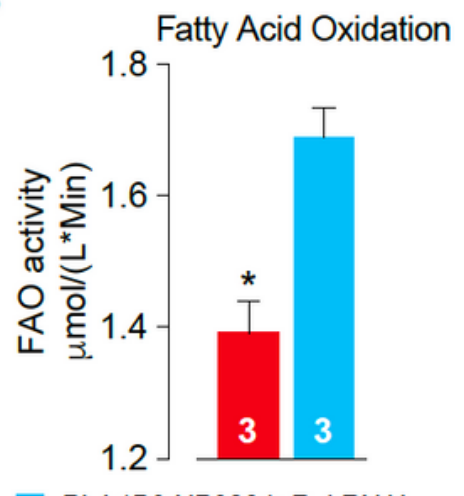

C

Pink1B9;NP3084>

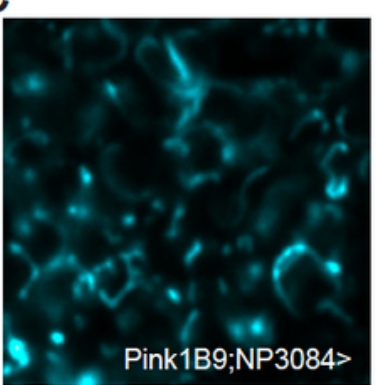

Pink1B9;NP3084>Rel RNAi
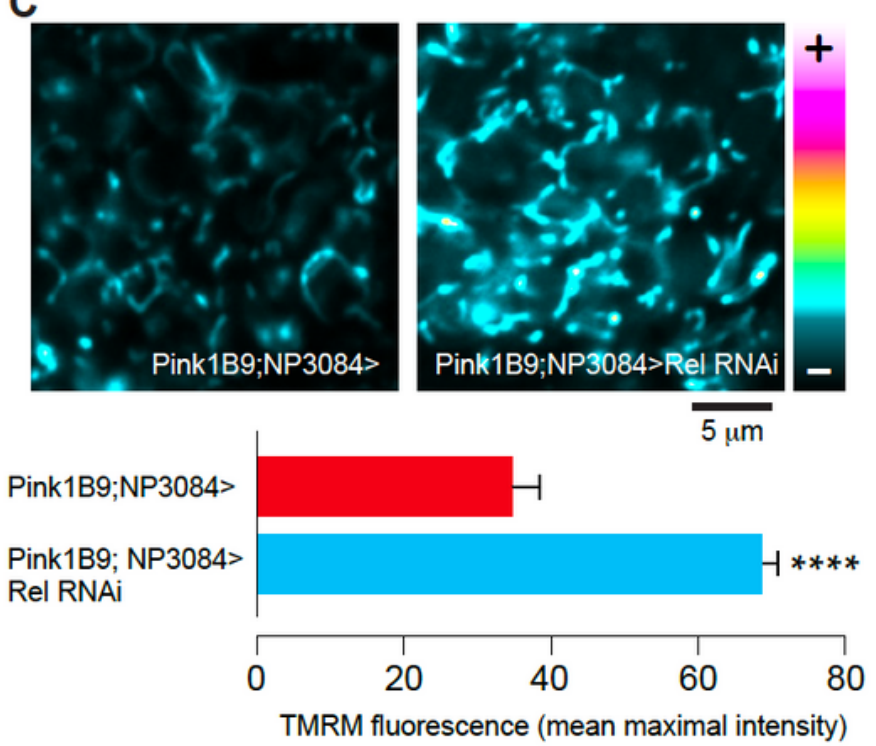

D

E

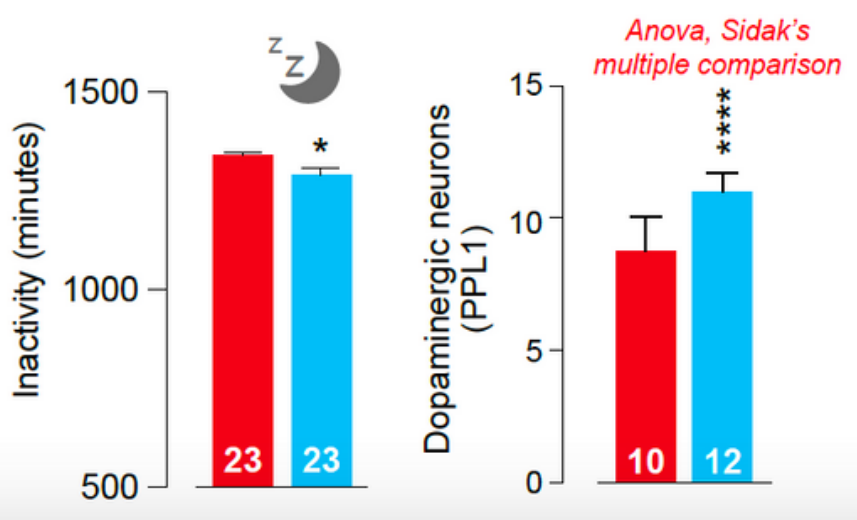

\section{Figure 7}

The suppression of Relish in the midgut of pink1-mutant flies rescues neurodegeneration. (A) The intestinal suppression of Relish reduces the levels of circulating triglycerides in pink1-mutant flies (means \pm SEMs; asterisks, two-tailed Student's t-test). (B) Fatty acid oxidation is increased by intestinal suppression of Relish (means \pm SDs; asterisks, one-way two-tailed Student's t-test). (C) Recovery of $\Delta \psi m$ following the intestinal suppression of Relish in pink1-mutant flies. Representative confocal images of 
whole mounted brains showing neurons loaded with TMRM are shown. The intensity levels of TMRM are visualized using a 5 -tone heat map. The data are shown as the means \pm SEMs ( $n=5$ per genotype;

asterisks, two-tailed unpaired t-test). (D) The intestinal suppression of Relish reduces the sleep defects of pink1-mutant flies (means \pm SEMs; asterisks, two-tailed Student's t-test). (E) The intestinal suppression of Relish prevents the loss of dopaminergic neurons in the PPL1 cluster of pink1-mutant flies (means \pm SDs; asterisks, one-way two-tailed Student's t-test). The genotypes in $(A)$ to $(E)$ are the following: Pink1B9; NP3084>: pink1B9; NP3084Gal4/+, Pink1B9; NP3084>Rel RNAi: pink1B9; NP3084Gal4/Rel KK109851.
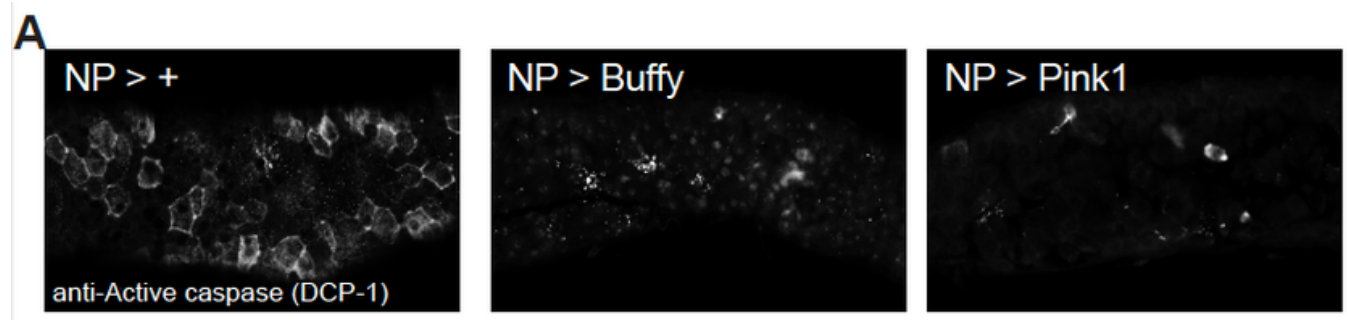

B
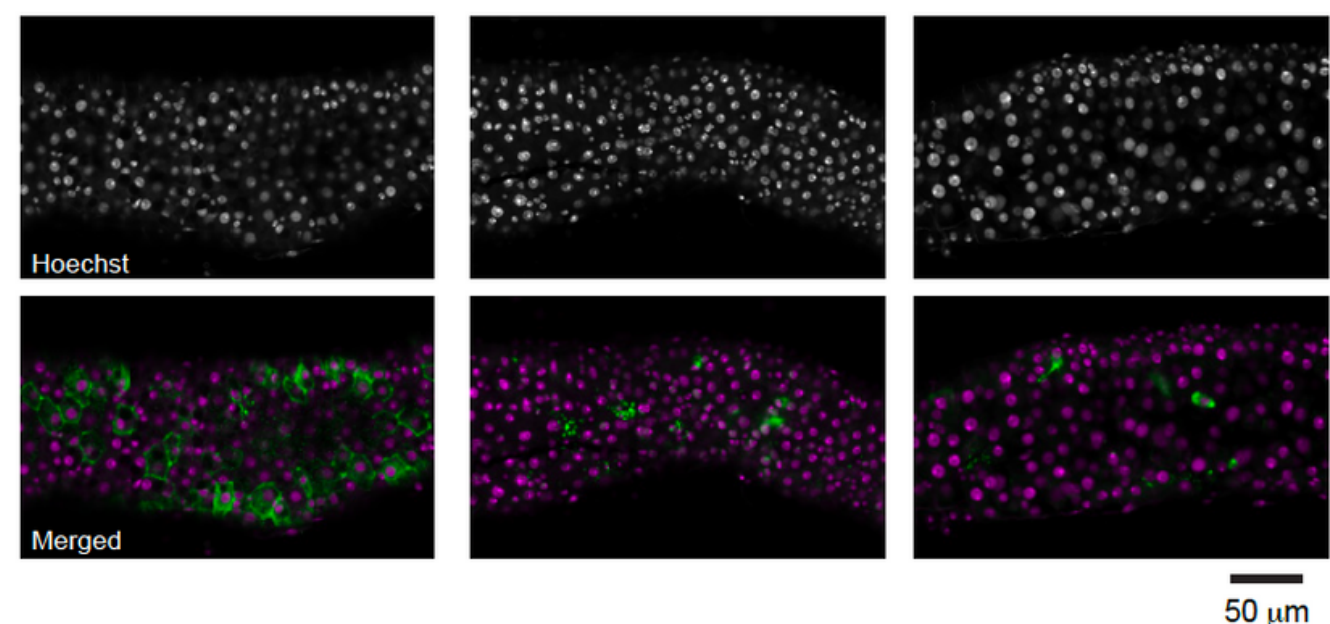

\section{Figure 8}

The blockage of intestinal cell death rescues neurodegeneration in pink1-mutant flies. (A and B) The intestinal expression of Buffy or Pink1 blocks apoptosis in the posterior midgut of pink1-mutant flies. (A) Representative confocal images of Dcp-1-positive cells. (B) The quantification of Dcp-1-positive cells in the posterior midgut shows that either Buffy or Pink1 reduces the number of Dcp-1-positive enterocytes (means \pm SDs; asterisks, one-way ANOVA with the Sidak multiple comparison test). (C) The intestinal expression of Buffy or Pink1 prevents the loss of dopaminergic neurons in the PPL1 cluster in pink1mutant flies (means \pm SDs; asterisks, one-way ANOVA with the Sidak multiple comparison test). The genotypes in (A) to (C) are the following: NP> +: pink1B9; NP3084Gal4/+; +, NP > Buffy: pink1B9; NP3084Gal4/UAS buffy; +, NP > Pink1; pink1B9; NP3084Gal4/UAS pink1.

\section{Supplementary Files}

This is a list of supplementary files associated with this preprint. Click to download.

- SupplementaryTable1.xlsx 
- SupplementaryTable2.xlsx

- SupplementaryTable3.xlsx

- SupplementaryTable4.xIsx 